\title{
Industrial and Mobile Robot Collision-Free Motion Planning Using Fuzzy Logic Algorithms
}

\author{
Tzafestas S.G. and Zavlangas P.
}

\section{Introduction}

Motion planning is a primary task in robot operation, where the objective is to determine collision-free paths for a robot that works in an environment that contains some moving obstacles (Latombe, 1991; Fugimura, 1991; Tzafestas, 1999). A moving obstacle may be a rigid object, or an object with joints such as an industrial manipulator. In a persistently changing and partially unpredictable environment, robot motion planning must be on line. The planner receives continuous flow of information about occurring events and generates new commands while previous planned motions are being executed. Off - line robot motion planning is a one - shot computation prior to the execution of any motion, and requires all pertinent data to be available in advance. With an automatic motion planner and appropriate sensing devices, robots can adapt quickly to unexpected changes in the environment and be tolerant to modeling errors of the workspace. A basic feature of intelligent robotic systems is the ability to perform autonomously a multitude of tasks without complete a priori information, while adapting to continuous changes in the working environment.

E Clearly, both robotic manipulators and mobile robots (as well their combina¿ tion, i.e. mobile manipulators (Seraji, 1998; Tzafestas \& Tzafestas, 2001)) need proper motion planning algorithms. For the robotic manipulators, motion $\frac{0}{0}$ planning is a critical aspect due to the fact that the end effector paths have al. have to follow a complex 3-dimensional path during the welding process. Specifying manually such paths can be tedious and time consuming. For the \& mobile robots (indoor and outdoor robots) motion planning and autonomous $\frac{\pi}{\pi}$ navigation is also a critical issue, as evidenced by applications such as office cleaning, cargo delivery, autonomous wheel chairs for the disabled,etc.

\& Our purpose in this chapter is to present a solution of the motion planner design problem using fuzzy logic and fuzzy reasoning. Firstly, the case of indus$\bar{\Phi}$ trial robotic manipulators is considered, and then the class of mobile robots is treated. The methodology adopted is primarily based on some recent results 
derived by the authors (Moustris \& Tzafestas, 2005; Zavlangas \& Tzafestas, $2000 ; 2001 ; 2002)$. To help the reader appreciate the importance of the techniques presented in the chapter, a short review is first included concerning the general robot motion planning problem along with the basic concepts and some recent results. A good promising practical approach is to use fuzzy logic along the path of behavior-based system design which employs Brooks' subsumption architecture (Brooks, 1986; Izumi \& Watanabe, 2000; Topalov \& Tzafestas, 2001; Watanabe et al., 1996; Watanabe et al., 2005).

Section 2 provides the overview of robot motion planning for industrial and mobile robots. Section 3 presents the authors' technique for industrial manipulators' fuzzy path planning and navigation. Section 4 extends this technique to mobile robots and discusses the integration of global and local path planning and navigation. Global path planning uses topological maps for representing the robot's environment at the global level, in conjunction with the potential field method. Section 5 presents a representative set of experimental results for the SCARA Adept 1 robotic manipulator and the Robuter III mobile robot (which can be equipped with a robotic arm). Results for both local and global path planning / navigation are included for the Robuter III robot. Finally, a general discussion on the results of the present technique is provided, along with some indications for future directions of research.

\section{Robot Motion Planning : An Overview}

\subsection{Review of Basic Motion Planning Concepts}

Robot motion planning techniques have received a great deal of attention over the last twenty years. It can roughly be divided into two categories : global and local. Most of the research in global techniques has been focused on off-line planning in static environments. A plan is then computed as a geometric path. An important concept developed by this research is the Configuration space or C-space of a robot (Latombe, 1991; Lozano-Perez, 1983).

The global techniques, such as road map (Latombe, 1991), cell decomposition (Latombe, 1991) and potential fields methods (Khatib, 1986), generally assume that a complete model of the robot's environment is available.

The roadmap approach to path planning consists of capturing the connectivity of the robot's free space in a network of one-dimensional curves (called the roadmap), lying in the free space. Once the roadmap has been constructed, it is used as a set of standardized paths. Path planning is thus reduced to connecting the initial and goal configuration to points in the roadmap and searching it for a path between these points (Latombe, 1991). 
Cell decomposition methods are perhaps the motion planning methods that have been most extensively studied so far (Latombe, 1991). They consist of decomposing the robot's free space into simple regions, called cells, such that a path between any two configurations in a cell can be easily generated. A nondirected graph representing the adjacency relation between the cells is then constructed and searched. This graph is called the connectivity graph. Its nodes are the cells extracted from the free space and two nodes are connected by a link if only the corresponding cells are adjacent. The outcome of the search is a sequence of cells called a channel. A continuous free path can be computed from this sequence (Latombe, 1991). A straightforward approach to motion planning is to discretize the configuration space into a fine regular grid of configurations and to search this grid for a free space. This approach requires powerful heuristics to guide the search. Several types of heuristics have been proposed. The most successful ones take the form of functions that are interpreted as potential fields (Latombe, 1991). The robot is represented as a point in configuration space, moving under the influence of an artificial potential produced by the goal configuration and the C-obstacles. Typically, the goal configuration generates an "attractive potential" which pulls the robot towards the goal, and the C-obstacles produce a "repulsive potential" which pushes the robot away from them. The generated gradient of the total potential is treated as an artificial force applied to the robot. At every configuration, the direction of this force is considered to be the most promising direction of motion.

The advantage of global approaches lies in the fact that a complete trajectory from the starting point to the target point can be computed off-line. However, global approaches are not appropriate for fast obstacle avoidance. Their strength is global path planning. Additionally, these methods were proven problematic when the global world model is inaccurate, or simply not available, as it is typically the case in the most populated environments. Some researchers have shown how to update global world models based on sensory inputs, using probabilistic representations. A second disadvantage of global methods is their low speed due to the inherent complexity of robot motion planning. This is particularly the case if the underlying world model changes with time, because of the resulting requirement for repeated adjustments of the global plan. In such cases, planning using a global model is usually too expensive to be done repeatedly.

Local approaches, on the other hand, use only a small fraction of the world model to generate robot control. This comes at the obvious disadvantage that they cannot produce optimal solutions. Local approaches are easily trapped at local minima. However, the key advantage of local techniques over global ones lies in their low computational complexity, which is particularly important when the world model is updated frequently based on sensor information. For example, potential field methods determine the next step by assuming that obstacles assert negative forces on the robot, and that the target location asserts a 
positive force. These methods are extremely fast, and they typically consider only a small subset of obstacles close to the robot. However, such methods have often failed to find trajectories between closely spaced obstacles; they also can produce oscillatory behaviour in narrow spaces.

\subsection{Motion Planning of Mobile Robots}

To be useful in the real world, mobile robots need to move safely in unstructured environments and achieve their given goals despite unexpected changes in their surroundings. The environments of real robots are rarely predictable or perfectly known so it does not make sense to make precise plans before moving. The robot navigation problem can be decomposed into the following two problems (Ratering \& Gini, 1995) :

- Getting to the goal. This is a global problem because short paths to the goal generally cannot be found using only local information. The topology of the space is important in finding good routes to the goal.

- Avoiding obstacles. This can often be solved using only local information, but for an unpredictable environment it cannot be solved in advance because the robot needs to sense the obstacles before it can be expected to avoid them.

Over the years, robot collision avoidance has been a component of high-level controls in hierarchical robot systems. Collision avoidance has been treated as a planning problem, and research in this area was focused on the development of collision-free path planning algorithms. These algorithms aim at providing the low-level control with a path that will enable the robot to accomplish its assigned task free from any risk of collision. However, this places limits on the robot's real-time capabilities for precise, fast, and highly interactive operations in a cluttered and evolving environment. Collision avoidance at the low-level control is not intended to replace high-level functions or to solve planning problems. The purpose is to make better use of low-level control capabilities in performing real-time operations. A number of different architectures for autonomous robot navigation have been proposed in the last twenty years (Latombe, 1991; Fugimura, 1991; Tzafestas, 1999). These include hierarchical architectures that partition the robot's functionalities into high-level (model and plan) and low-level (sense and execute) layers; behaviour - based architectures that achieve complex behaviour by combining several simple behaviourproducing units; and hybrid architectures that combine a layered organization with a behaviour-based decomposition of the execution layer (see e.g., (Izumi \& Watanabe, 2000; Watanabe et al., 1996; Topalov \& Tzafestas, 2001; Watanabe et al., 2005; Lozano-Perez, 1983; Khatib, 1986; Ratering \& Gini, 1995; Erdmann \& Lozano-Perez, 1987; Griswold \& Elan, 1990; Gil de Lamadrid \& Gini, 1990; 
Fibry, 1987; Gat, 1991; Sugeno \& Nishida, 1985; Yen \& Pflunger, 1992)). While the use of hybrid architectures is gaining increasing consensus in the field, a number of technological gaps still remain.

As mentioned in Section 2.1, the classical approaches can be mainly divided into two categories : global path planning and local navigation (Latombe, 1991). The global approaches preassume that a complete representation of the configuration space has been computed before looking for a path. They are complete in the sense that if a path exists it will be found. Unfortunately, computing the complete configuration space is very time consuming, worst, the complexity of this task grows exponentially as the number of degrees of freedom increases. Consequently, today most of the robot path planners are used off-line. The planner is equipped with a model of the environment and produces a path which is passed to the robot controller for execution. In general, the time necessary to achieve this, is not short enough to allow the robot to move in dynamic environments. The local approaches need only partial knowledge of the robot's workspace. The decisions to move the robot are taken using local criteria and heuristics to choose the most promising direction. Consequently, the local methods are much faster. Unfortunately, they are not complete, it may happen that a solution exists and cannot be found. The local approaches consider planning as an optimization problem, where finding a path to the goal configuration corresponds to the optimization of some given function. As an optimization technique, the local approaches are subject to get trapped in some local minima, where a path to the goal has not been found and from which it is impossible or, at least, very difficult to escape.

From the above, it is very clear, that both global and local techniques have many advantages, as well as important disadvantages. The output of a global path planner is a continuous path along which the robot will not collide with obstacles. However, any model of the real world will be incomplete and inaccurate, thus collisions may still occur if the robot moves blindly along such a path. One conventional application is for the robot to track the global path. More recently, work has been done on increasing the level of competence, by including real-time collision avoidance capabilities. Such local or reactive behaviours operate in real time but cannot solve the global problem of moving to an arbitrary goal. It is very clear that to built a complete system, the above approaches must be combined. A path planner must provide the robot with a global path to the goal. A local controller then moves the robot along the global path while handling small changes in the environment and unexpected or moving obstacles.

Some researchers have solved the navigation problem by solving these two sub-problems one after the other. A path is first found from the robot's initial position to the goal and then the robot approximates this path as it avoids obstacles. This method is restrictive in that the robot is required to stay fairly close to or perhaps on a given path. This would not work well if the path goes 
through a passageway which turns out to be blocked by an unforeseen obstacle. Solutions that are only local or reactive (Brooks, 1986) can lead the robot into local minima traps. Solutions that assume a priori knowledge of the position of the obstacles (e.g. (Fugimura, 1991; Erdmann \& Lozano-Perez, 1987)), or select a path using only information on stationary obstacles, and determine the speed of the robot while following the path (e.g. (Griswold \& Elan, 1990)), or solutions that require the robot to stay within some distance from its assigned path, while avoiding unknown moving obstacles (e.g., (Gil de Lamadrid \& Gini, 1990)), are not always sufficiently flexible to deal with situations in which an obstacle blocks a path to the goal.

In the general case, knowledge of the environment is partial and approximate; sensing is noisy; the dynamics of the environment can only be partially predicted; and robot's hardware execution is not completely reliable. Though, the robot needs to make decisions and execute actions at the time-scale of the environment. Classical planning approaches have been criticized for not being able to adequately cope with this situation, and a number of reactive approaches to robot control have been proposed (e.g. (Fibry, 1987; Gat, 1991)), including the use of fuzzy control techniques (e.g., (Martinez et al., 1994; Seraji \& Howard, 2002; Sugeno \& Nishida, 1985; Yen \& Pflunger, 1992)). Reactivity provides immediate response to unpredicted environmental situations by giving up the idea of reasoning about future consequences of actions. Reasoning about future consequences (sometimes called "strategic planning"), however, is still needed in order to intelligently solve complex tasks.

Some recent developments in mobile robot navigation using fuzzy logic algorithms include (Parhi, 2005) and (El Hajjaji, 2004). In (Parhi, 2005) the fuzzy controller enables the robot to avoid obstacles that are not mobile robots. The fuzzy rules steer the robot according to whether there are obstacles or targets around it and how far they are from it. Fuzzy logic is suitable for this problem because this information is usually not precisely known. In (El Hajjaji, 2004) the case of 4-wheel automotive vehicles is considered which are modeled by a Takagi-Sugeno type of model. The fuzzy controller is then designed to improve the stability of the vehicle. A comprehensive study of the kinematics of nonholonomic mobile manipulators composed by an $n_{a}$-joint robotic arm and a nonholonomic mobile platform having two independently driven wheels is provided in (Bayle et al., 2003). Finally, a new approach to the navigation of mobile robots for dynamic obstacle avoidance is proposed in (Belkhous et al., 2005). This approach merges the static and dynamic modes of path planning to provide an algorithm giving fast optimal solutions for static environments, and produces a new path whenever an unanticipated situation occurs. 
3. Fuzzy Path Planning and Navigation of Industrial Manipulators

\subsection{Fuzzy Obstacle Avoidance}

Here, we will outline a technique developed by the authors (Zavlangas \& Tzafestas, 2000), which has been primarily influenced by Khatib's (1986) artificial potential field method and the subsumption architecture developed by Brooks (1986). The local navigation approach is chosen because our main goal is to develop an on-line planner for fast collision - free trajectory generation. The proposed local navigator was implemented and applied to several practical scenarios. Our experimental results, some of which will be presented in Section 5, are very satisfactory. The technique is based on separate fuzzy logicbased obstacle avoidance units, each controlling one individual link $l_{j}$, $j=1, \ldots, n$ Each unit has two principal inputs :

1. the distance between the link and the nearest obstacle $d_{j}$, and

2. the difference between the current link configuration and the target configuration,

$\theta_{j}-\theta_{j, \text { target }}=\Delta \theta_{j}$

The output variable of each unit is the motor command $\tau_{j}$. All these variables can be positive or negative, i.e., they inform both about the magnitude and the sign of displacement relative to the link - left or right. The motor command which can be interpreted as an actuation for the link motor is fed to the manipulator at each iteration (Figure 1). For the calculation of the distance, the only obstacles considered are those which fall into a bounded area surrounding each link and move along with it. In this implementation, each such area is chosen to be a cylindrical volume around each link. The area is as long as the link and reaches up to a predefined horizon. This area can be seen as a simplified model for the space scanned by ranging sensors (for example, ultra-sonic sensors) attached to the sides of a link (Pedrycz, 1995). Of course, other shapes to describe the 3-dimensional scan areas are conceivable. It is, for example, advisable to deal with the blind zones near the joints when the magnitude of the angles is large so as to assure that small moving obstacles are not missed by the algorithm (Pedrycz, 1995). 


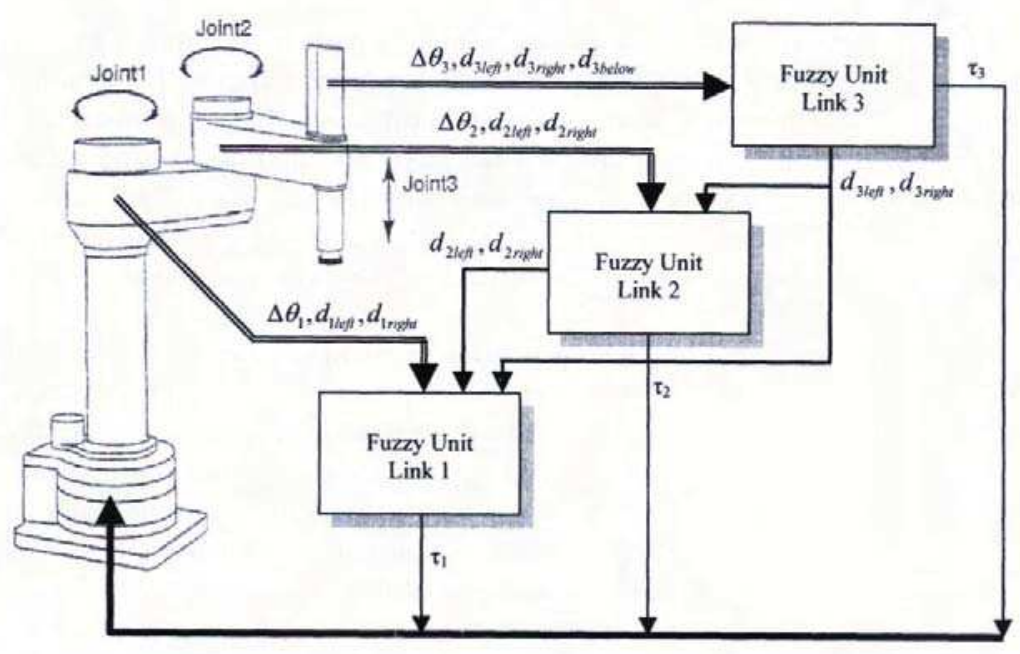

Figure 1. The Adept 1 industrial robotic manipulator connected to the corresponding fuzzy units.

Each fuzzy unit receives via an input the difference between target and actual configuration, and, via a second input, two values in a sequential way representing the distance between the corresponding link and the nearest obstacle on the left and on the right of this link. If no obstacle is detected inside the scan area, the fuzzy unit is informed of an obstacle in the far distance. Additionally, proximal units are informed about obstacles in the vicinity of more distal links. Besides an input from ultrasonic sensors, a camera can be used to acquire the environment. Either a stationary camera or a set of cameras which oversee the entire workspace can be utilised (Pedrycz, 1995; Jaitly \& Fraser, 1996). The task of each fuzzy unit is to provide a control function which produces an appropriate motor command from the given inputs. In broad lines, the control function can be described as follows : on the one hand the function has to lead the corresponding link to its attracting end - position; on the other hand, it has to force the link to back up when approaching an obstacle which conveys a repelling influence. The fuzzy-rule-base (which represents the control function in each fuzzy unit) is built up by using common sense rules.

In our particular implementation, at each iteration the distances of the nearest obstacle on the left $\left(d_{\text {jleft }}\right)$ and on the right $\left(d_{\text {jright }}\right)$ of link $l_{j}$ are fed sequentially into the fuzzy unit. This process could also be carried out in a parallel fashion where two equivalent fuzzy controllers compute the response for the left and the right obstacle separately. The resulting two motor commands are superimposed, hence, both obstacles influence the final motor command which is applied to the link. The use of this method guarantees that the repulsion caused by one obstacle on one side of the link does not result in a collision with a nearby obstacle on the opposite side. Only those obstacles are considered which are the nearest on the left and right. 
In addition, fuzzy units of distal links communicate information about the distance to their nearest obstacles on the left and right to units of more proximal links. Once sent to fuzzy units of more proximal links, this information can be used by the decision process of those units to slow down or even reserve the motion of the more proximal links. Without this propagation of information the control strategy might fail in situations where one obstacle is "known" only to a fuzzy unit of a distal link, while proximal links continue their motion based on their local environment dictating an adverse motion for the rest of the arm. This is especially important, since the same change of angle occurring at a proximal link and at a distal link produces a different velocity at the manipulator's tip. Thus, the motion of a proximal link might not be sufficiently compensated by an adverse motion at a more distal link. Fuzzy units are only fed with the distance values of those obstacles which are inside the scan range. If no obstacle is detected inside a scan range, the fuzzy unit is informed of an obstacle which is far left or far right, respectively.

\subsection{The Fuzzy Navigation Algorithm}

The first input of each fuzzy unit is the difference between the actual angle and the target angle, $\theta_{j}-\theta_{j, \text { target }}=\Delta \theta_{j} \in \Theta_{j}, j=1, \ldots, n$ ( $n$ is the number of links). The value $\Delta \theta_{j}$ is positive if the target is on the right, and negative if the target is on the left. The second input receives values describing the distance between link $l_{j}$ and the nearest obstacles on the left and right in the "scanned" region, $d_{j} \in D_{j}$. An obstacle on the left produces a negative distance value, while an obstacle on the right produces a positive one. The single output is the motor command $\tau_{j} \in T_{j}$. A positive motor command moves the link to the left and a negative one to the right (Althoefer, 1996; Althoefer \& Fraser, 1996).

Each universe of discourse $D_{j}$ can be partitioned by fuzzy sets $\mu_{1}^{(j)}, \ldots, \mu_{p j}^{(j)}$. Each of the sets $\mu_{\tilde{p} j}^{(j)}, \tilde{p}_{j}=1, \ldots, p_{j}$, represents a mapping $\mu_{\tilde{p} j}^{(j)}\left(d_{j}\right): D_{j} \rightarrow[0,1]$ by which $d_{j}$ is associated with a number in the interval $[0,1]$ indicating to what degree $d_{j}$ is a member of the fuzzy set. Since $d_{j}$ is a signed value, "close_left", for example, may be considered as a particular fuzzy value of the variable distance and each $d_{j}$ is assigned a number $\mu_{\text {close_left }}\left(d_{j}\right) \in[0,1]$ which indicates the extent to which that $d_{j}$ is considered to be close_left (Mamdani \& Assilian, 1981). In an equivalent way, fuzzy sets $v_{1}^{(j)}, \ldots, v_{q j}^{(j)}$ can be defined over the universe of discourse $\Theta_{j}$. The Fuzzy Navigator is based on the Mamdani fuzzy model (Mamdani, 1974) and has an output set which is partitioned into fuzzy sets $\tau_{\tilde{r} j}$

There is a variety of functions that can be employed to represent fuzzy sets (Mamdani \& Assilian, 1981). In the proposed controller asymmetrical trape- 
zoidal functions were employed to represent the fuzzy sets. The parameters, $m l^{(j)}, m r^{(j)}$, which are the x-coordinates of the left and right zero crossing, respectively, and $\mathrm{mcl}^{(j)}, \mathrm{mcr}^{(j)}$, which describe the x-coordinate (left and right) where the fuzzy set becomes 1, define the following trapezoidal functions :

$$
\mu_{p_{j}}^{(j)}\left(d_{j}\right)= \begin{cases}\min \left(\frac{\left(d_{j}-m l_{p_{j}}^{(j)}\right)}{\left(m c l_{p_{j}}^{(j)}-m l_{p_{j}}^{(j)}\right)}, 0\right) & \text { if } m l_{p_{j}}^{(j)}<d_{j} \leq m c l_{p_{j}}^{(j)}, \\ 1 & \text { if } m c l_{p_{j}}^{(j)}<d_{j} \leq m c r_{p_{j}}^{(j)}, \\ \min \left(\frac{\left(d_{j}-m r_{p_{j}}^{(j)}\right)}{m c r_{p_{j}}^{(j)}-m r_{p_{j}}^{(j)}}, 0\right) & \text { if } m c r_{p_{j}}^{(j)}<d_{j} \leq m r_{p_{j}}^{(j)} .\end{cases}
$$

As commonly done, the trapezoidal functions are continued as constant values of magnitude 1 at the left and right side of the interval (Equations (2) and (3)) :

$$
\mu_{p_{j}}^{(j)}\left(d_{j}\right)=\left\{\begin{array}{lr}
1 & \text { if } m l_{1}^{(j)}<d_{j} \leq m c l_{1}^{(j)}, \\
1 & \text { if } m c l_{1}^{(j)}<d_{j} \leq m c r_{1}^{(j)}, \\
\min \left(\frac{\left(d_{j}-m r_{1}^{(j)}\right)}{\left(m c r_{1}^{(j)}-m r_{1}^{(j)}\right)}, 0\right) & \text { if } m c r_{1}^{(j)}<d_{j} \leq m r_{1}^{(j)} .
\end{array}\right.
$$

and

$$
\mu_{p_{j}}^{(j)}\left(d_{j}\right)= \begin{cases}\min \left(\frac{\left(d_{j}-m l_{p_{j}}^{(j)}\right)}{\left(m c l_{p_{j}}^{(j)}-m l_{p_{j}}^{(j)}\right)}, 0\right) & \text { if } m l_{p_{j}}^{(j)}<d_{j} \leq m c l_{p_{j}}^{(j)}, \\ 1 & \text { if } m c l_{p_{j}}^{(j)}<d_{j} \leq m c r_{p_{j}}^{(j)}, \\ 1 & \text { if } m c r_{p_{j}}^{(j)}<d_{j} \leq m r_{p_{j}}^{(j)} .\end{cases}
$$

Note, that for the two fuzzy sets of Equations (2) and (3) :

$$
m c r_{p_{j}}^{(j)}=m r_{p_{j}}^{(j)}, m c l_{p_{j}}^{(j)}=m l_{p_{j}}^{(j)}
$$


The fuzzy sets for $\Delta \theta_{j}$ can be defined in the same way. The fuzzy sets $m_{1}^{(j)}, \ldots, m_{p}^{(j)}$ and $n_{1}^{(j)}, \ldots, n_{q}^{(j)}$ for link 2 as functions of the two inputs $d_{j}$ and $\Delta \theta_{j}$ are shown in Figure 2, together with the output fuzzy set.

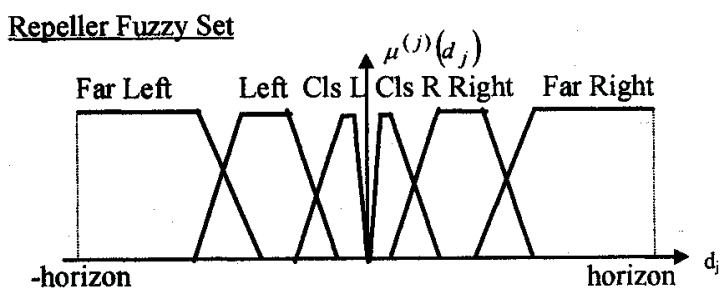

(a)

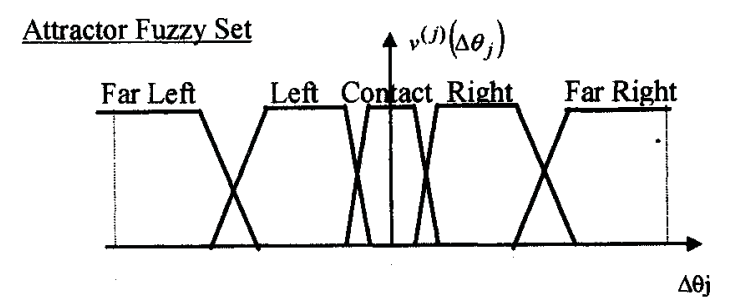

(b)

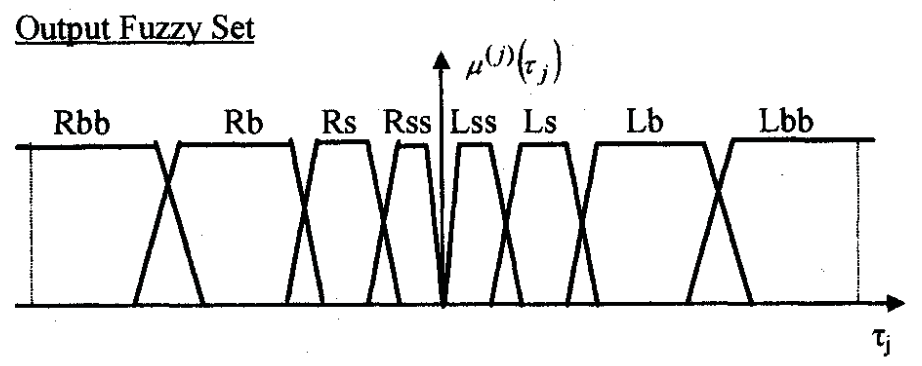

Figure 2. Repeller, attractor and output fuzzy sets for link 2.

The first diagram (a) is the fuzzy set of the distance between the link and the obstacle, and the second one (b) is the fuzzy set of the difference between the actual and target configuration. The output fuzzy set is shown in the third diagram (c).

Additionally to the two inputs $d_{j}$ and $\Delta \theta_{j}$, each fuzzy unit (apart from the most distal one) uses the distance fuzzy sets of more distal links $\mu_{\tilde{p}_{j+1}}^{(j+1)}, \ldots, \mu_{\tilde{p}_{n}}^{(n)}$ for decision making to assure that proximal links are slowed down, in case a more distal link is about to collide with an obstacle. Thus, each unit uses the following fuzzy sets : $\mu_{\tilde{p}_{k}}^{(k)}, k=j+1, \ldots, n$, and $v_{\tilde{q}_{j}}^{(j)}$. Each of the fuzzy sets $\mu_{\tilde{p}_{k}}^{(k)}$ and $v_{\tilde{q}_{j}}^{(j)}$ are associated with linguistic terms $A_{\tilde{p}_{j}}^{(j)}$ and $B_{\tilde{q}_{j}}^{(j)}$, respectively. Thus, for link $l_{j}$ the linguistic control rules $R_{1}^{(j)}, \ldots, R_{r_{j}}^{(j)}$, which constitute the rule base, can be defined as : 
$R_{\tilde{r}_{j}}^{(j)}: I F d_{j}$ is $A_{\tilde{p}_{j}}^{(j)} A N D \ldots A N D d_{n}$ is $A_{\tilde{p}_{n}}^{(n)} A N D \Delta \theta_{j}$ is $B_{\tilde{q}_{j}}^{(j)}$ THEN $\tau_{\tilde{r}_{j}}$

where $\tilde{r}_{j}=1, \ldots, r_{j}, r_{j}$, is the number of rules for the fuzzy unit of link $l_{j}$, and $\tau_{\tilde{r}_{j}}$ is a numerical entry in the rule base used in the defuzzification process (Equation (5)). The most popular methods to calculate the fuzzy intersection (fuzzy - AND) are the minimum and product operators (Tzafestas \& Venetsanopoulos, 1994; Zadeh, 1965; 1973). If the minimum operator is used, the minimum of the inputs is chosen. If the product operator is chosen, the inputs are multiplied with each other. While the result of the first approach contains only one piece of information, the second approach produces results which are influenced by all inputs (Brown, 1994).

Here, the fuzzy intersection is calculated by using the product operator " $*$ " :

$$
\sigma_{\tilde{r}_{j}}=\mu_{\tilde{p}_{j}, \tilde{r}_{j}}^{(j)}\left(d_{j}\right) \bigcap \cdots \bigcap \mu_{\tilde{p}_{n}, \tilde{r}_{n}}^{(n)}\left(d_{n}\right) \bigcap v_{\tilde{q}_{j}, \tilde{r}_{j}}^{(j)}\left(\Delta \theta_{j}\right)=\mu_{\tilde{p}_{j}, \tilde{r}_{j}}^{(j)}\left(d_{j}\right) * \ldots * \mu_{\tilde{p}_{n}, \tilde{r}_{n}}^{(n)}\left(d_{n}\right) * v_{\tilde{q}_{j}, \tilde{r}_{j}}^{(j)}\left(\Delta \theta_{j}\right)
$$

The output of the unit is given by the centroid defuzzification over all rules (Kosko, 1992) :

$$
\tau_{j}=\frac{\sum_{r_{j}=1}^{r_{j}} \tau_{r_{j}} * \mu_{p_{j}, r_{j}}^{(j)}\left(t_{r_{j}}\right)}{\sum_{r_{j}=1}^{r_{j}} \mu_{p_{j}, r_{j}}^{(j)}\left(t_{r_{j}}\right)}
$$

The fuzzy rule base for link 2 is displayed in Table I.

\begin{tabular}{|lllllll|}
\hline & $\begin{array}{l}\text { Far } \\
\text { left }\end{array}$ & Left & $\begin{array}{l}\text { Close } \\
\text { left }\end{array}$ & $\begin{array}{l}\text { Close } \\
\text { right }\end{array}$ & Right & $\begin{array}{l}\text { Far } \\
\text { right }\end{array}$ \\
\hline Far left & Ls & Rs & Rbb & Lb & Ls & Ls \\
Left & Lss & Rs & Rbb & Ls & Lss & Lss \\
Contact & nil & nil & nil & nil & nil & nil \\
Right & Rss & Rss & Rs & Lbb & Ls & Rss \\
Far right & Rs & Rs & Rb & Lbb & Ls & Rs \\
\hline Lss, Rss : very small to the left / right & & \\
Ls, Rs : small to the left / right & & & \\
Lb, Rb : big to the left / right & & & \\
Lbb, Rbb : very big to the left / right & & \\
\hline
\end{tabular}

Table 1. FAM matrix (Fuzzy Associative Memory) for link 2 


\section{Fuzzy Path Planning and Navigation of Mobile Robots}

\subsection{General Description}

Basically, the technique to be described for mobile fuzzy path planning and navigation is the same with that described in Section 3 for the case of industrial manipulators, i.e. it is based on Khatib's potential field method (Khatib, 1986) and on Brooks subsumption structure (Brooks, 1986).

Khatib computes an artificial potential field that has a strong repelling force in the vicinity of obstacles and an attracting force produced by the target location. The superposition of the two forces creates a potential field, which incorporates information about the environment. Following the steepest gradient from a start position, a path can be found that guides the robot to the target position avoiding obstacles. In our approach the amount of computation, that is required, is reduced by using only the nearest obstacles to determine the direction of motion. The main idea of Brooks is a collection of modules, which are interconnected on different layers with different hierarchies. These modules are for example wall following, obstacle avoidance, goal reaching, etc. Depending on sensory input, a module becomes active and generates a command for the robot. While Brooks' system resembles an expert system where for any input signal one specific reaction module or a specific combination of modules is active, our fuzzy approach is a parallel processing strategy where each input contributes to the final decision (see Section 3.2).

The technique is based on two fuzzy - based controllers, one for steering control, and the other for velocity control. The steering controller has three principal inputs :

1) the distance between the robot and the nearest obstacle $d_{j}$,

2) the angle between the robot and the nearest obstacle $\gamma_{j}$, and

$3)$ the angle between the robot's direction and the straight line connecting the current position of the robot and the goal configuration $\theta_{j}=\alpha_{j}-\beta_{j}$, where $\beta_{j}$ is the angular difference between the straight line connecting the robot's current position and the goal configuration, and $\alpha_{j}$ is the current direction of the robot (see Fig. 3).

The output variable of this unit is the required change of angle $\Delta \theta_{j}$, and can be considered as a command for the robot's steering actuators. The velocity controller has two principal inputs :

1) the distance between the robot and the nearest obstacle $d_{j}$,

2) the distance between the robot and the goal configuration $d_{g_{j}}$. 
The output variable of this unit is an acceleration command $\Delta v_{j}$, and can be considered as a command for the robot's drive actuators. All these variables can be positive or negative, i.e. they do not only inform about the magnitude, but also about the sign of displacement relative to the robot - left or right. The motor commands are fed to the mobile platform at each iteration.

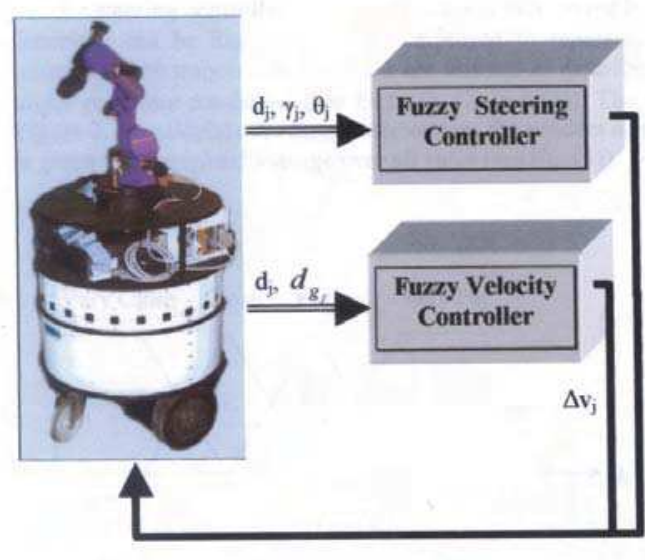

(a)

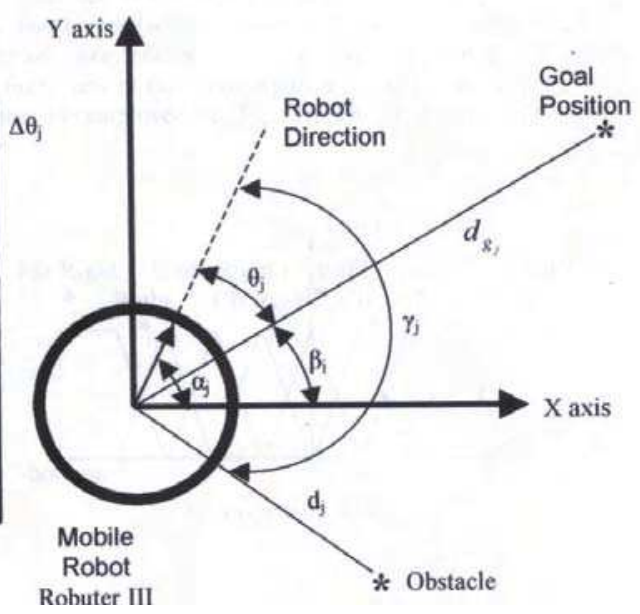

(b)

Figure3. (a) The Robosoft Robuter III mobile robot of IRAL / NTUA connected to the corresponding fuzzy-based obstacle avoidance unit.

This steering control unit receives via an input the angle between the robot's direction and the straight line connecting the current position of the robot and the goal configuration $\theta_{j}=\alpha_{j}-\beta_{j}$, and the distance and the angle to the nearest obstacle $\left(d_{j}, \gamma_{j}\right)$ (see (b)). If no obstacle is detected inside the scan area, the fuzzy unit is informed of an obstacle in the far distance. The output variable of this unit is the required change of angle $\Delta \theta_{j}$, and can be considered as a command for the robot's steering actuators. The velocity control unit has two inputs : the distance between the robot and the nearest obstacle $d_{j}$, and the distance between the robot and the goal configuration $d_{g_{j}}$. The output variable of this unit is an acceleration command $\Delta v_{j}$ and can be considered as a command for the robot's driving actuators.

The obstacles considered are those that fall into a confined area surrounding the robot and moving along with it. Here, this area is chosen to be a cylindrical volume around the mobile platform. This area is regarded as a simplified model for the space scanned by ranging sensors (for example ultrasonic sensors) attached to the sides of the robot (Pedrycz, 1995). Besides an input from ultrasonic sensors, a camera can also be used to acquire the environment. Mo- 
bile robots are usually equipped with a pan / tilt platform where a camera is mounted. This camera can be utilized as shown in (Pedrycz, 1995; Jaitly et al., 1996; Kamon \& Rivlin, 1997). If no obstacle is detected inside the scan area, the fuzzy units are informed of an obstacle in the far distance. The task of the fuzzy units is to provide a control function, that produces appropriate motor commands from the given inputs. This control function on the one hand has to lead the mobile robot to its attracting goal-position, and on the other hand it has to force the robot to back up when approaching an obstacle which conveys a repelling influence. The fuzzy-rule - base (which represents the control function in the fuzzy unit) is constructed using common sense rules or by a neural network training algorithm (see e.g., (Tzafestas \& Zavlangas, 1999)). An alternative fuzzy motion control scheme of mobile robots which employs the sliding - mode control principle can be found in (Rigatos \& Tzafestas, 2000).

\subsection{Detailed Description}

As mentioned in Section 4.1 the proposed methodology is based on two fuzzy - based controllers, one for steering control, and the other for velocity control. The fuzzy controllers here are based on the functional reasoning control principles developed by Sugeno (see, for example, (Sugeno, 1985; Sugeno \& Murakami, 1984)). For the steering controller, each input space is partitioned by fuzzy sets as shown in Fig. 4. Here, asymmetrical triangular and trapezoidal functions are utilized to describe each fuzzy set, which allow a fast computation, essential under real - time conditions (see Eqs. (6) - (8) and (11)). The fuzzy sets of the three inputs $d_{j}, \gamma_{j}$ and $\theta_{j}$ are depicted in Figure 4 . To calculate the fuzzy intersection, the product operator is employed (see Eq (10)). The final output of the unit is given by a weighted average over all rules (see Eq. (11)). Intuitively, the rules for obstacle - avoiding navigation can be written as sentences with three antecedents and one conclusion. This structure lends itself to a tabular representation such as the one shown in Table 2. This table represents the prior knowledge of the problem domain. The tools of fuzzy logic allow us to translate this intuitive knowledge into a control system. To translate Table 2 into fuzzy logic, the universe of discourse $D_{j}$ which describes the distance $d_{j} \in D_{j}$ to the obstacle is partitioned by fuzzy sets $\mu_{1}^{(j)}, \ldots, \mu_{p_{j}}^{(j)}$, where $p_{j}$ is the number of fuzzy sets. Each set $\mu_{\tilde{p}_{j}}^{(j)}, \tilde{p}_{j}=1, \ldots, p_{j}$, represents a mapping $\mu_{\tilde{p}_{j}}^{(j)}\left(d_{j}\right): D_{j} \rightarrow[0,1]$ by which $d_{j}$ is considered as a particular fuzzy value of the variable distance and each $d_{j}$ is associated with, e.g., a number in the interval $[0,1]$ indicating to what degree $d_{j}$ is a member of the fuzzy set. Since $d_{j}$ is a measure of distance, "very_close", it may be assigned a number : $\mu_{\text {very_close }}\left(d_{j}\right) \in[0,1]$ which indicates the extent to which this particular $d_{j}$ is considered to be "very_close" (Mamdani \& Assilian, 1981). 


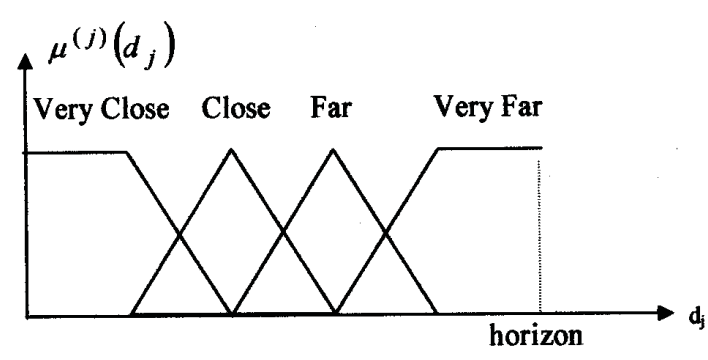

(a) Distance to Obstacle

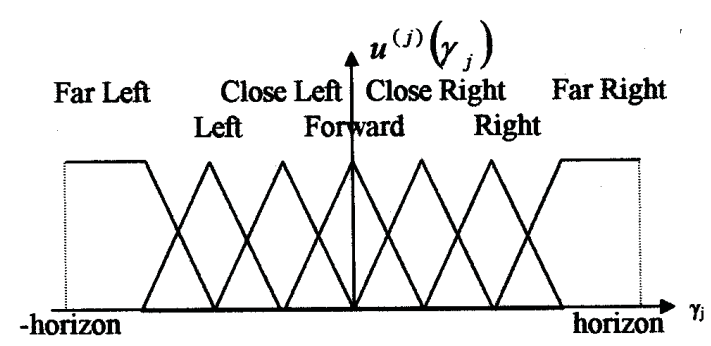

(c) Angle to Obstacle

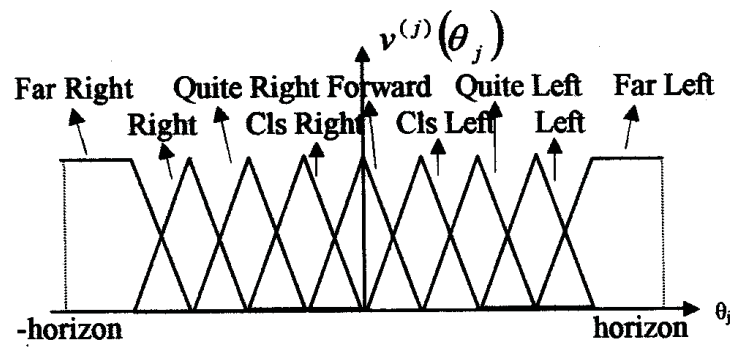

(b) Angle to Goal Position

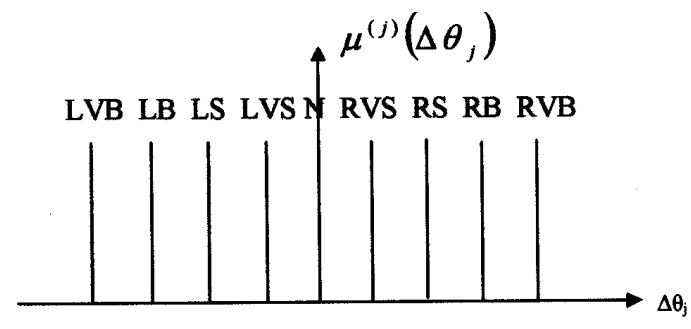

(d) Steering Motor Command

Fig. 4. Fuzzy sets for the mobile robot: (a) distance to obstacle, (b) angle between robot and goal position, (c) angle between robot and obstacle, and (d) steering motor command. Note that the output is not partitioned into fuzzy sets, but consists of crisp values.

\begin{tabular}{|c|c|c|c|c|}
\hline$v_{j} / \mu_{j}$ & very close & close & far & very far \\
\hline far right & right big & right small & left very big & left very big \\
\hline right & right big & right small & left big & left big \\
\hline quite right & right big & right small & left small & left small \\
\hline close right & right big & right small & left very small & left very small \\
\hline forward & null & null & null & null \\
\hline close left & $\begin{array}{l}\text { right } \\
\text { small }\end{array}$ & $\begin{array}{l}\text { right } \\
\text { small }\end{array}$ & $\begin{array}{l}\text { right } \\
\text { small }\end{array}$ & $\begin{array}{l}\text { right } \\
\text { small }\end{array}$ \\
\hline quite left & right small & right small & right small & right small \\
\hline left & right big & right big & right big & right big \\
\hline far left & right very big & right very big & right very big & right very big \\
\hline \multicolumn{5}{|c|}{$\begin{array}{l}\text { This rule-base is a translation of the common - sense knowledge of the problem do- } \\
\text { main into the language of fuzzy logic. Rows represent the fuzzy measures of the dis- } \\
\text { tance to an obstacle, while columns are fuzzy representations of the angle to the goal. } \\
\text { Each element of the table can be interpreted as a particular motor actuation com- } \\
\text { mand. }\end{array}$} \\
\hline
\end{tabular}

Table 2. A rule - base for the mobile robot when $\gamma_{j}$ is "far_left". 
Similarly, fuzzy sets $v_{1}^{(j)}, \ldots v_{q_{j}}^{(j)}$ can be defined over the universe of discourse $\Theta_{j}$ which represents the angle between the robot's direction and the straight line connecting the current position of the robot and the goal configuration : $\theta_{j} \in \Theta_{j}$. Finally, fuzzy sets $u_{1}^{(j)}, \ldots, u_{g_{j}}^{(j)}$ can be defined over the universe of discourse $\Gamma_{j}$ that represents the angle to the nearest obstacle $\gamma_{j} \in \Gamma_{j}$. In contrast to the Mamdani's controller, Sugeno's controller (see (Sugeno, 1985; Sugeno \& Murakami, 1984; Tzafestas \& Zikidis, 2001)), of which ours is an example, has an output set which is not partitioned into fuzzy sets (see Fig. 2). Thus, the rule conclusions merely consist of scalars $\Delta \theta_{\tilde{r}_{j}}, \tilde{r}_{j}=1, \ldots, r_{j}$.

The fuzzy sets $\mu_{\tilde{p}_{j}}^{(j)}, \tilde{p}_{j}=1, \ldots, p_{j}$, are described by asymmetrical triangular and trapezoidal functions. Defining the parameters, $m l_{\tilde{p}_{j}}^{(j)}$ and $m r_{\tilde{p}_{j}}^{(j)}$ as the $\mathrm{x}$-coordinates of the left and right zero crossing respectively, and $m c l_{\tilde{p}_{j}}^{(j)}$ and $m c r_{\tilde{p}_{j}}^{(j)}$ as the $x$-co-ordinates of the left and right side of the trapezoid's plateau, the trapezoidal functions can be written as :

$\mu_{\tilde{p}_{j}}^{(j)}\left(d_{j}\right)=\left\{\begin{array}{lc}\max \left(\left(d_{j}-m l_{\tilde{p}_{j}}^{(j)}\right) /\left(m c l_{\tilde{p}_{j}}^{(j)}-m l_{\tilde{p}_{j}}^{(j)}\right), 0\right) & \text { if } d_{j}<m c l_{\tilde{p}_{j}}^{(j)} \\ 1 & \text { if } m c l_{\tilde{p}_{j}}^{(j)} \leq d_{j} \leq m c r_{\tilde{p}_{j}}^{(j)} \\ \max \left(\left(d_{j}-m r_{\tilde{p}_{j}}^{(j)}\right) /\left(m c r_{\tilde{p}_{j}}^{(j)}-m r_{\tilde{p}_{j}}^{(j)}\right), 0\right) & \text { if } d_{j}>m c r_{\tilde{p}_{j}}^{(j)}\end{array}\right.$

with $\tilde{p}_{j}=1, \ldots, p_{j}$. Triangular functions can be achieved by setting $m c l_{\tilde{p}_{j}}^{(j)}=m c r_{\tilde{p}_{j}}^{(j)}$.

At the left and right sides of the interval, the functions are continued as constant values of magnitude one, i.e. :

$\mu_{1}^{(j)}\left(d_{j}\right)= \begin{cases}1 & \text { if } d_{j} \leq m c r_{1}^{(j)} \\ \max \left(\left(d_{j}-m r_{1}^{(j)}\right) /\left(m c r_{1}^{(j)}-m r_{1}^{(j)}\right), 0\right) & \text { if } d_{j}>m c r_{1}^{(j)}\end{cases}$

and

$\mu_{p_{j}}^{(j)}\left(d_{j}\right)= \begin{cases}\max \left(\left(d_{j}-m l_{p_{j}}^{(j)}\right) /\left(m c l_{p_{j}}^{(j)}-m l_{p_{j}}^{(j)}\right), 0\right) & \text { if } d_{j} \leq m c l_{p_{j}}^{(j)} \\ 1 & \text { if } d_{j}>m c l_{p_{j}}^{(j)}\end{cases}$

The fuzzy sets for $\theta_{j}$ and $\gamma_{j}$ are defined analogously. Figure 4 shows the fuzzy sets $\mu_{1}^{(j)}, \ldots, \mu_{p_{j}}^{(j)}, v_{1}^{(j)}, \ldots, v_{q_{j}}^{(j)}$ and $u_{1}^{(j)}, \ldots, u_{g_{j}}^{(j)}$. 
Every fuzzy set, $\mu_{\tilde{p}_{j}}^{(j)}, v_{\tilde{q}_{j}}^{(j)}$ and $u_{\tilde{g}_{j}}^{(j)}$, is associated with linguistic terms $A_{\tilde{q}_{j}}^{(j)}, B_{\tilde{q}_{j}}^{(j)}$ and $C_{\tilde{g}_{j}}^{(j)}$ respectively. Thus, for the mobile robot, the linguistic control rules $R_{1}^{(j)}, \ldots, R_{r_{j}}^{(j)}$ which constitute the rule base can be defined as :

$R_{\tilde{r}_{j}}^{(j)}: \mathbf{I F} d_{j}$ is $A_{\tilde{p}_{j}}^{(j)}$ AND $\theta_{j}$ is $B_{\tilde{q}_{j}}^{(j)}$ AND $\gamma_{j}$ is $C_{\tilde{g}_{j}}^{(j)}$ THEN $f\left(\tau_{\tilde{r}_{j}}\right) \quad\left(\tilde{r}_{j}=1, \ldots, r_{j}\right)$

where the AND operations use the t-norm product operator :

$\sigma_{\tilde{r}_{j}}=\mu_{\tilde{p}_{j}, \tilde{r}_{j}}^{(j)}\left(d_{j}\right) \cap v_{\tilde{q}_{j}, \tilde{r}_{j}}^{(j)}\left(\theta_{j}\right) \cap u_{\tilde{g}_{j}, \tilde{r}_{j}}^{(j)}\left(\gamma_{j}\right)=\mu_{\tilde{p}_{j}, \tilde{r}_{j}}^{(j)}\left(d_{j}\right) * v_{\tilde{q}_{j}, \tilde{r}_{j}}^{(j)}\left(\theta_{j}\right) * u_{\tilde{g}_{j}, \tilde{r}_{j}}^{(j)}\left(\gamma_{j}\right)$

Finally, the output of the unit is given by a weighted average over all rules (see Fig. 2 and (Topalov \& Tzafestas, 2001)) :

$\Delta \theta_{j}=\frac{\sum_{\tilde{r}_{j}=1}^{r_{j}} \sigma_{\tilde{r}_{j}} \cdot \Delta \theta_{\tilde{r}_{j}}}{\sum_{\tilde{r}_{j}=1}^{r_{j}} \sigma_{\tilde{r}_{j}}}$

Eq. (9) together with Eqs. (10) and (11) define how to translate the intuitive knowledge reflected in Table 2 into a fuzzy rule - base. The details of this translation can be modified by changing the number of fuzzy sets, the shape of the sets (by choosing the parameters, $m l_{\tilde{p}_{j}}^{(j)}$ and $m r_{\tilde{p}_{j}}^{(j)}, m c l_{\tilde{p}_{j}}^{(j)}, m c r_{\tilde{p}_{j}}^{(j)}$ as well as the value $\Delta \theta_{\tilde{r}_{j}}$ of each of the rules in Eq. (11). A technique for the automated selection of the above parameters is provided in (Tzafestas \& Zikidis, 2001). As an example, the control rules for the particular mobile robot are shown in Table 2. In this application, the number of fuzzy sets which fuzzify the obstacle distance $d_{j}$, the angle to the goal $\theta_{j}$ and the angle to the nearest obstacle $\gamma_{j}$ are chosen to be four, nine and seven, respectively. All the other parameters were refined by trial and error. 


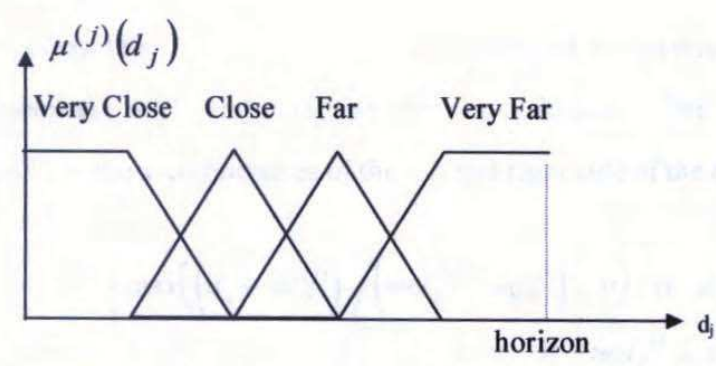

(a) Distance to Obstacle

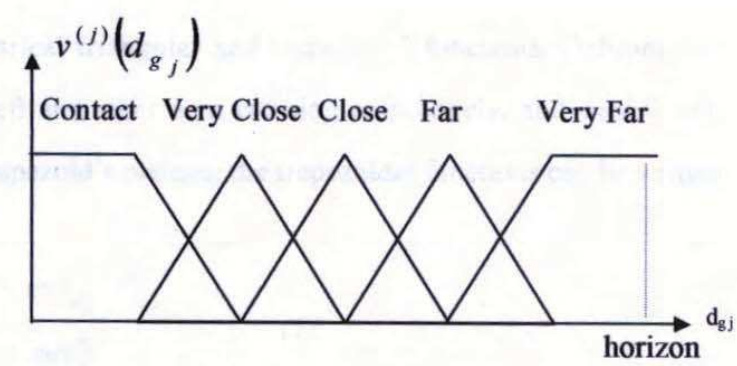

(b) Angle to goal configuration

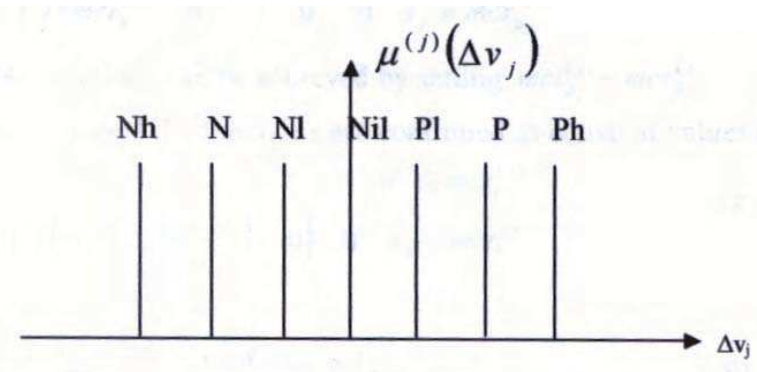

(c) Drive Motor Command

Figure 5. Fuzzy sets for the velocity control unit of the mobile robot : (a) the distance between the robot and the nearest obstacle, and (b) the distance between the robot and the goal configuration. Note that the output is an acceleration command and is not partitioned into fuzzy sets, but consists of crisp values.

For the velocity controller, each input space is partitioned by fuzzy sets as shown in Figure 5. Asymmetrical triangular and trapezoidal functions have also been used to describe each fuzzy set, which allow a fast computation, essential under real-time conditions (see Eqs. (12) - (13)).

To calculate the fuzzy intersection, the product operator is employed (see Eq. (16)). The final output of the unit is given by a weighted average over all rules (see Eq. (17)).

Intuitively, the rules for the velocity controller can be written as sentences with two antecedents and one conclusion. This structure is represented by Table 3 which provides the prior knowledge of the problem domain. The tools of fuzzy logic allow us to translate this intuitive knowledge into a control system. 


\begin{tabular}{|l|l|l|l|l|}
\hline$v\left(d_{g_{j}}\right) / \mu\left(d_{j}\right)$ & very close & close & far & very far \\
\hline contact & negative high & negative high & negative high & negative high \\
\hline very close & negative high & negative high & negative & negative low \\
\hline close & negative high & negative & nil & nil \\
\hline far & negative high & negative & positive low & positive \\
\hline very far & negative high & negative & positive & positive high \\
\hline $\begin{array}{l}\text { Rows represent the fuzzy measures of the distance to an obstacle, while columns } \\
\text { are fuzzy representations of the distance between the robot and the goal configu- } \\
\text { ration. Each element of the table can be interpreted as a particular drive motor } \\
\text { actuation command for the acceleration and deceleration of the mobile robot. }\end{array}$
\end{tabular}

Table 3. The rule - base for the velocity controller.

The fuzzy sets $\mu_{\tilde{p}_{j}}^{(j)}, \tilde{p}_{j}=1, \ldots, p_{j}$, are described by asymmetrical triangular and trapezoidal functions. Defining the parameters, $m l_{\tilde{p}_{j}}^{(j)}$ and $m r_{\tilde{p}_{j}}^{(j)}$ as the $\mathrm{x}$-coordinates of the left and right zero crossing respectively, and $m c l_{\tilde{p}_{j}}^{(j)}$ and $m c r_{\tilde{p}_{j}}^{(j)}$ as the x-co-ordinates of the left and right side of the trapezoid's plateau, the trapezoidal functions can be written as :

$\mu_{\tilde{p}_{j}}^{(j)}\left(d_{j}\right)=\left\{\begin{array}{lc}\max \left(\left(d_{j}-m l_{\tilde{p}_{j}}^{(j)}\right) /\left(m c l_{\tilde{p}_{j}}^{(j)}-m l_{\tilde{p}_{j}}^{(j)}\right), 0\right) & \text { if } d_{j}<m c l_{\tilde{p}_{j}}^{(j)} \\ 1 & \text { if } m c l_{\tilde{p}_{j}}^{(j)} \leq d_{j} \leq m c r_{\tilde{p}_{j}}^{(j)} \\ \max \left(\left(d_{j}-m r_{\tilde{p}_{j}}^{(j)}\right) /\left(m c r_{\tilde{p}_{j}}^{(j)}-m r_{\tilde{p}_{j}}^{(j)}\right), 0\right) & \text { if } d_{j}>m c r_{\tilde{p}_{j}}^{(j)}\end{array}\right.$

with $\tilde{p}_{j}=1, \ldots, p_{j}$. Triangular functions can be achieved by setting $m c l_{\tilde{p}_{j}}^{(j)}=m c r_{\tilde{p}_{j}}^{(j)}$.

At the left and right side of the interval the functions are continued as constant values of magnitude one :

$\mu_{1}^{(j)}\left(d_{j}\right)= \begin{cases}1 & \text { if } d_{j} \leq m c r_{1}^{(j)} \\ \max \left(\left(d_{j}-m r_{1}^{(j)}\right) /\left(m c r_{1}^{(j)}-m r_{1}^{(j)}\right), 0\right) & \text { if } d_{j}>m c r_{1}^{(j)}\end{cases}$

and

$\mu_{p_{j}}^{(j)}\left(d_{j}\right)= \begin{cases}\max \left(\left(d_{j}-m l_{p_{j}}^{(j)}\right) /\left(m c l_{p_{j}}^{(j)}-m l_{p_{j}}^{(j)}\right), 0\right) & \text { if } d_{j} \leq m c l_{p_{j}}^{(j)} \\ 1 & \text { if } d_{j}>m c l_{p_{j}}^{(j)}\end{cases}$ 
The fuzzy sets for $d_{g j}$ are defined analogously. Figure 5 shows the fuzzy sets $\mu_{1}^{(j)}, \ldots, \mu_{p_{j}}^{(j)}$ and $v_{1}^{(j)}, \ldots, v_{q_{j}}^{(j)}$.

The linguistic control rules $R_{1}^{(j)}, \ldots, R_{r_{j}}^{(j)}$, which constitute the rule base, are defined as :

$R_{\tilde{r}_{j}}^{(j)}:$ IF $d_{j}$ is $A_{\tilde{p}_{j}}^{(j)}$ AND $d_{g j}$ is $B_{\tilde{q}_{j}}^{(j)}$ THEN $f\left(\Delta v_{\tilde{r}_{j}}\right) \quad\left(\tilde{r}_{j}=1, \ldots, r\right)$

where the AND operations use the t-norm product operator, defined as :

$\sigma_{\tilde{r}_{j}}=\mu_{\tilde{p}_{j}, \tilde{r}_{j}}^{(j)}\left(d_{j}\right) \cap v_{\tilde{q}_{j}, \tilde{r}_{j}}^{(j)}\left(d_{g_{j}}\right)=\mu_{\tilde{p}_{j}, \tilde{r}_{j}}^{(j)}\left(d_{j}\right) * v_{\tilde{q}_{j}, \tilde{r}_{j}}^{(j)}\left(d_{g_{j}}\right)$

Finally, the output of the velocity control unit is given by a weighted average over all rules (Eq. 16) :

$\Delta v_{j}=\frac{\sum_{\tilde{r}_{j}=1}^{r_{j}} \sigma_{\tilde{r}_{j}} \cdot \Delta v_{\tilde{r}_{j}}}{\sum_{\tilde{r}_{j}=1}^{r_{j}} \sigma_{\widetilde{r}_{j}}}$

\subsection{Integrated global and local path planning}

The path planner and navigator presented in Sections 4.1 and 4.2 is of the reactive type and is used at the local level. The full path following problem involves also a global path planner at a higher level (called geometric level) as shown in Fig. 6. The authors have derived and implemented a global path planner based on the potential field method which was selected for its mathematical elegance and simplicity. However, as it is evident, more complex / complete global path planning techniques can be embedded into our system. Global path planning strategies are more easily planned using a topological map, where the planner can decide the sequence of rooms and corridors to be traversed (Dudek et al., 1991; Ryu \& Yang, 1999; Kortenkamp \& Weymouth, 1994; Thrun, 1999; Kuipers \& Levitt, 1988). 


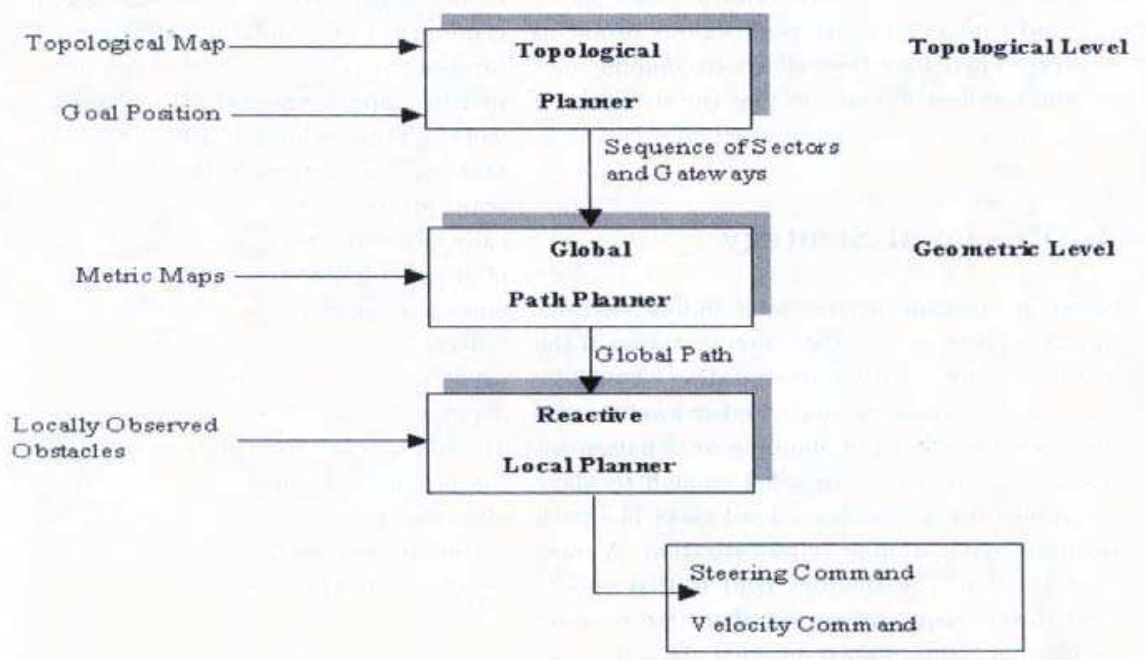

Figure 6. Three - level path planning and navigation.

The topological maps (networks) are placed at the top level of the hierarchical structure. The authors have conducted a number of experiments where both the topological and local metric maps were given to the robot a priori. The enormous compactness of topological maps when compared to the underlying grid-based map, increases substantially the efficiency of global planning. The paths are planned using the abstract topological map of the robot's environment (Fig. 7). Shortest paths on the topological maps can be easily found using one of the standard graph search algorithms, such as Dijkstra's or Floyd and Warshal's shortest path algorithm, the $A^{*}$ algorithm, or dynamic programming. In our case we have used the Dijkstra's shortest path algorithm. This algorithm finds the best (shortest) path on the topological map going from the sector that contains the current location of the robot to the one which contains the goal. For example, a plan to get from the "start" position in Fig. 7 to an office desk in room $R_{2}$ may be :

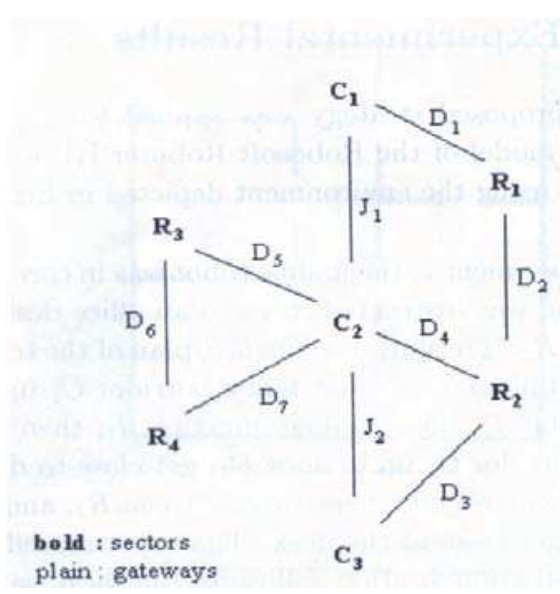

(a) 


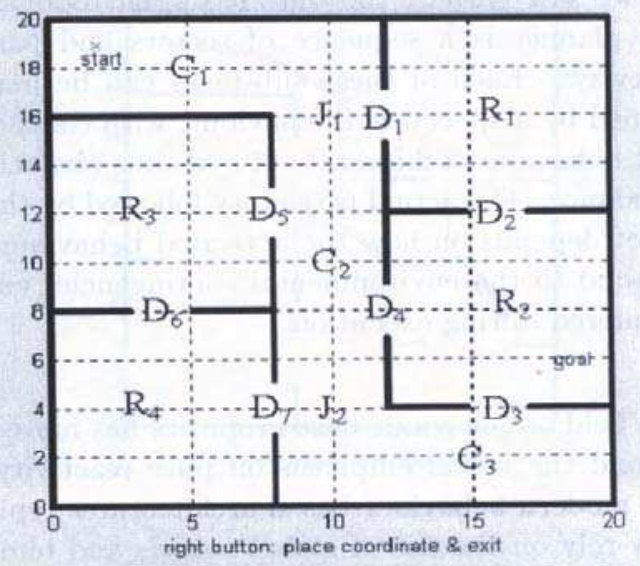

(b)

Figure 7. (a) The topological map used for the experiments, and (b) the corresponding network of the local metric maps.

\section{Experimental Results}

\subsection{Industrial Manipulator}

To test the functionality and performance of the basic algorithms, the fuzzy navigator was applied to a simulated 3-dof industrial manipulator (Adept 1). All experiments were carried out on a personal computer (Pentium II, 350 $\mathrm{MHz}$ ).

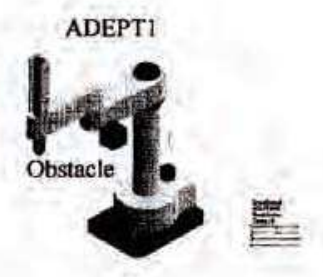

(1)

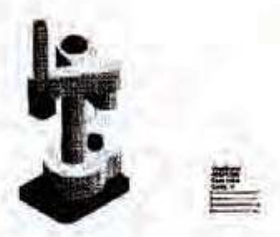

(4)

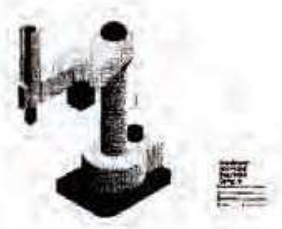

(2)

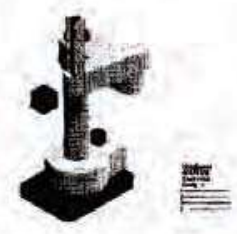

(5)

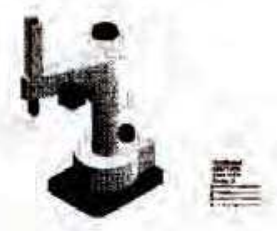

(3)

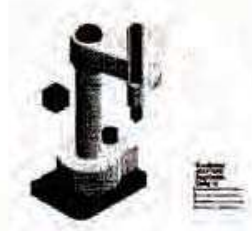

(6) 
(a)

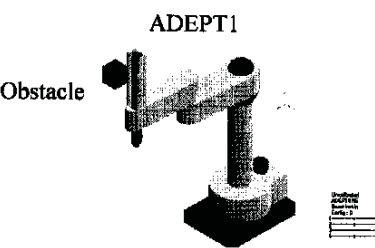

(1)

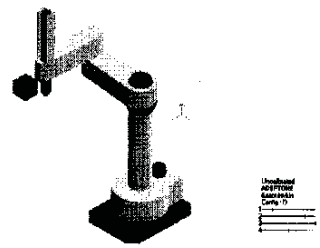

(4)

(b)

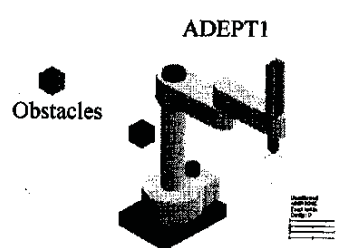

(1)

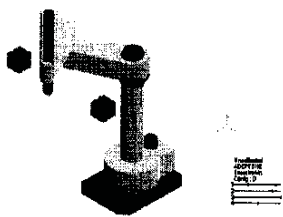

(c)

(4)

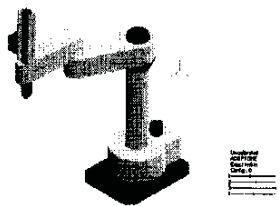

(2)

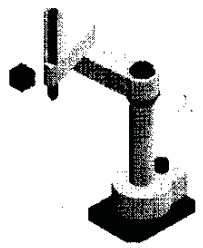

(5)

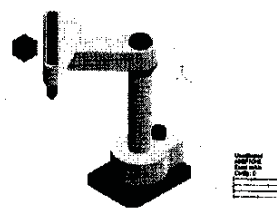

(3)

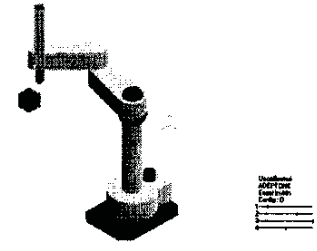

(6)

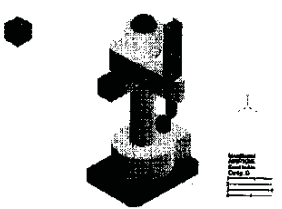

(2)

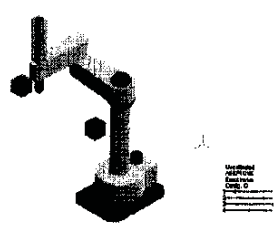

(5)

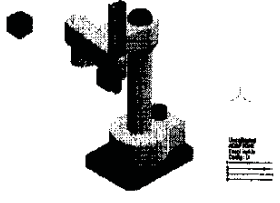

(3)

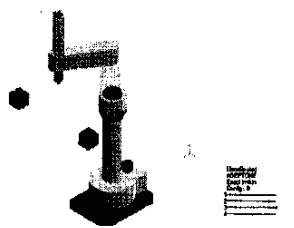

(6)

Figure 8. Simulation results using the ADEPT 1 industrial manipulator.

The proposed fuzzy navigator was successfully tested for the path planning / obstacle avoidance problem in three working scenarios with different obstacle constellations (a), (b) and (c), each time providing the manipulator with a collision - free trajectory.

The fuzzy navigator was developed using Microsoft $C$ and for the visualization and animation of the robot's path Workspace 4 software package was used.

The performance of the fuzzy navigator for the Adept 1 industrial manipulator was tested in a variety of environments, with different obstacle constellations and working scenarios, even in dynamic environments with moving obstacles. In all cases, the fuzzy navigator provided the system with a collision free motion. Simulation results obtained in three different working scenarios (with different obstacle constellations) are presented in Figure 8. 


\subsection{Mobile Robot local path planning}

The experimental results were first derived for the simulated omnidirectional mobile robot of the IRAL Laboratory (Robosoft Robuter III) using a Personal Computer (Pentium IV, 1.3 GHz) and the Matlab 5.2 software package. The fuzzy navigator was tested in a variety of environment scenarios (with both static and moving obstacles). In all cases a collision - free motion was obtained. A representative set of results are shown in Fig. 9 for four distinct working scenarios.

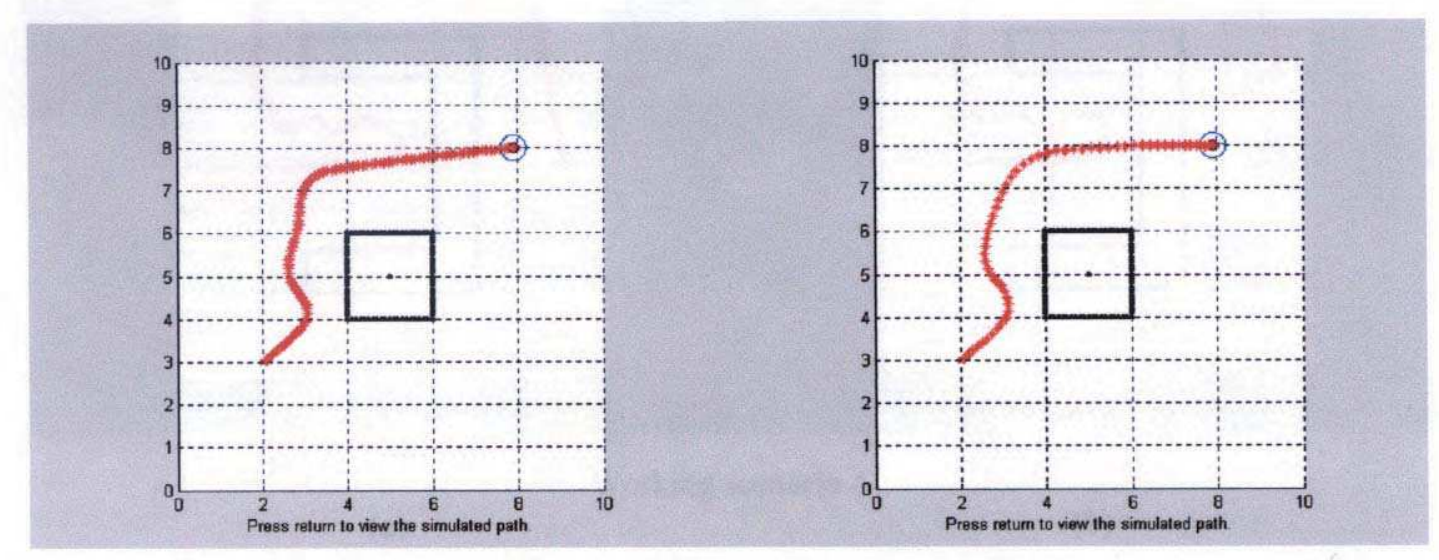

(a)

Working scenario 1

(b)

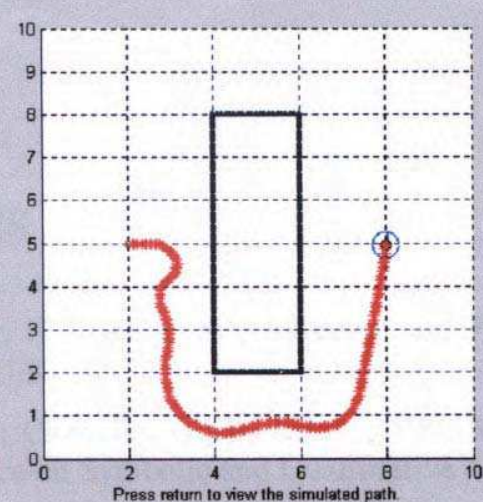

(a)

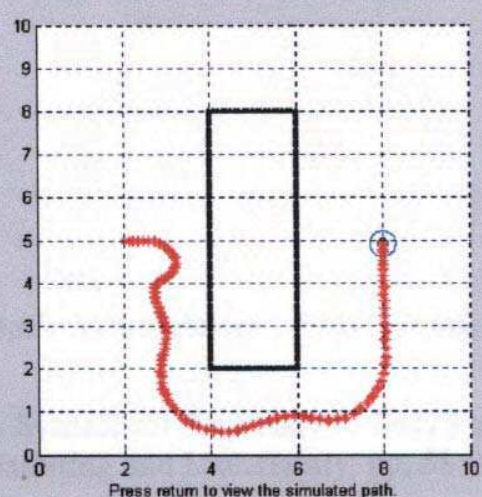

Working scenario 2

(b) 

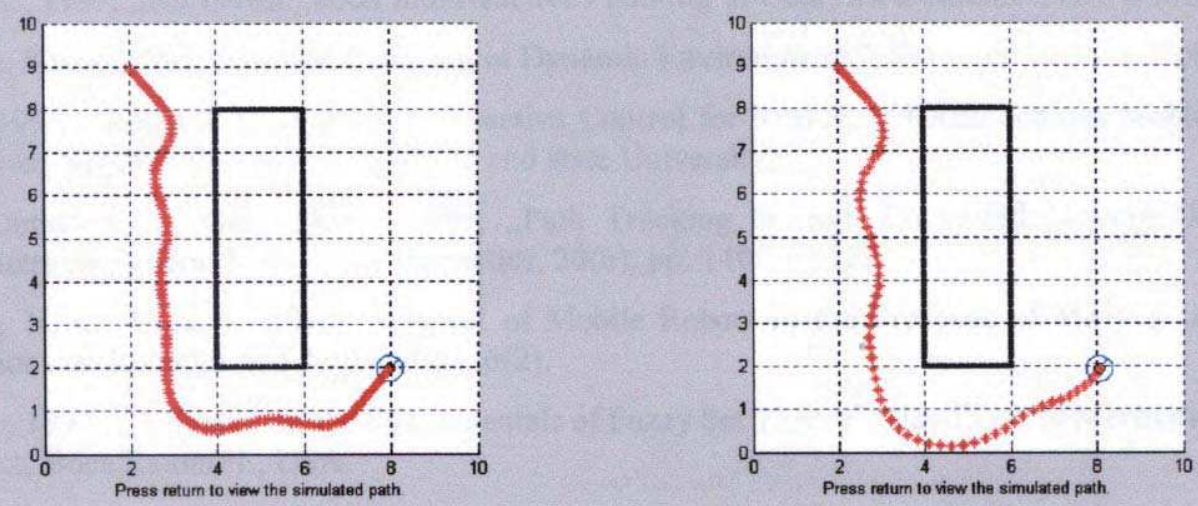

(a)

Working scenario 3

(b)
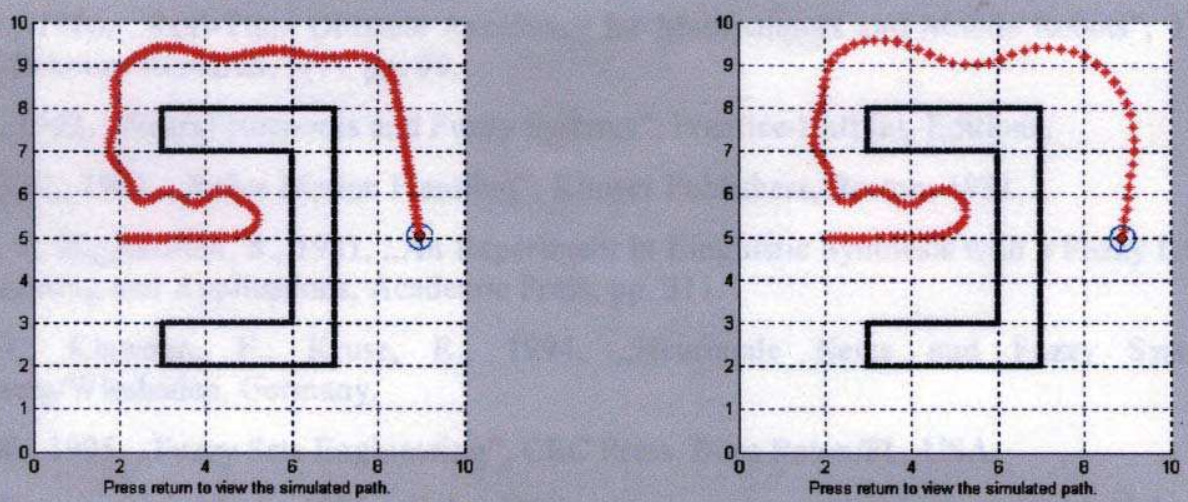

(a)

Working scenario 4

(b)

Figure 9. Simulation results using the Robosoft Robuter III mobile robot.

The proposed strategy was successfully tested in four working scenarios with different obstacle constellations each time providing the mobile robot with a collision - free movement. In (a) the path of the robot with only steering control is shown, and in (b) the path with the steering and velocity controllers is shown.

\subsection{Mobile robot global path planning}

The topological - map based global path planning technique was applied to the simulated Robosoft Robuter III mobile robot using the environment depicted in Fig. 7.

In experiment 1 , the mobile robot was in corridor $C_{1}$ and was instructed to go to an office desk in room $R_{2}$. The output sequence / plan of the topological 
planner is : first follow corridor $C_{1}$ up to junction $J_{1}$, then traverse junction $J_{1}$, then follow corridor $C_{2}$ up to door $D_{4}$, get-close-to door $D_{4}$, then cross it, then traverse room $R_{2}$, and finally get-close-to the desk. The robot moved to its goal configuration following the local paths generated by the path planner.

In experiment 2, the mobile robot was in room $R_{1}$ and was instructed to go to a position in corridor $C_{1}$. The output sequence / plan of the topological planner is : first traverse room $R_{1}$ up to door $D_{1}$, get-close-to door $D_{1}$, then cross it, then reverse junction $J_{1}$, then follow corridor $C_{1}$ and finally get-close-to goal position. The robot moved to its goal configuration following the local paths generated by the path planner.
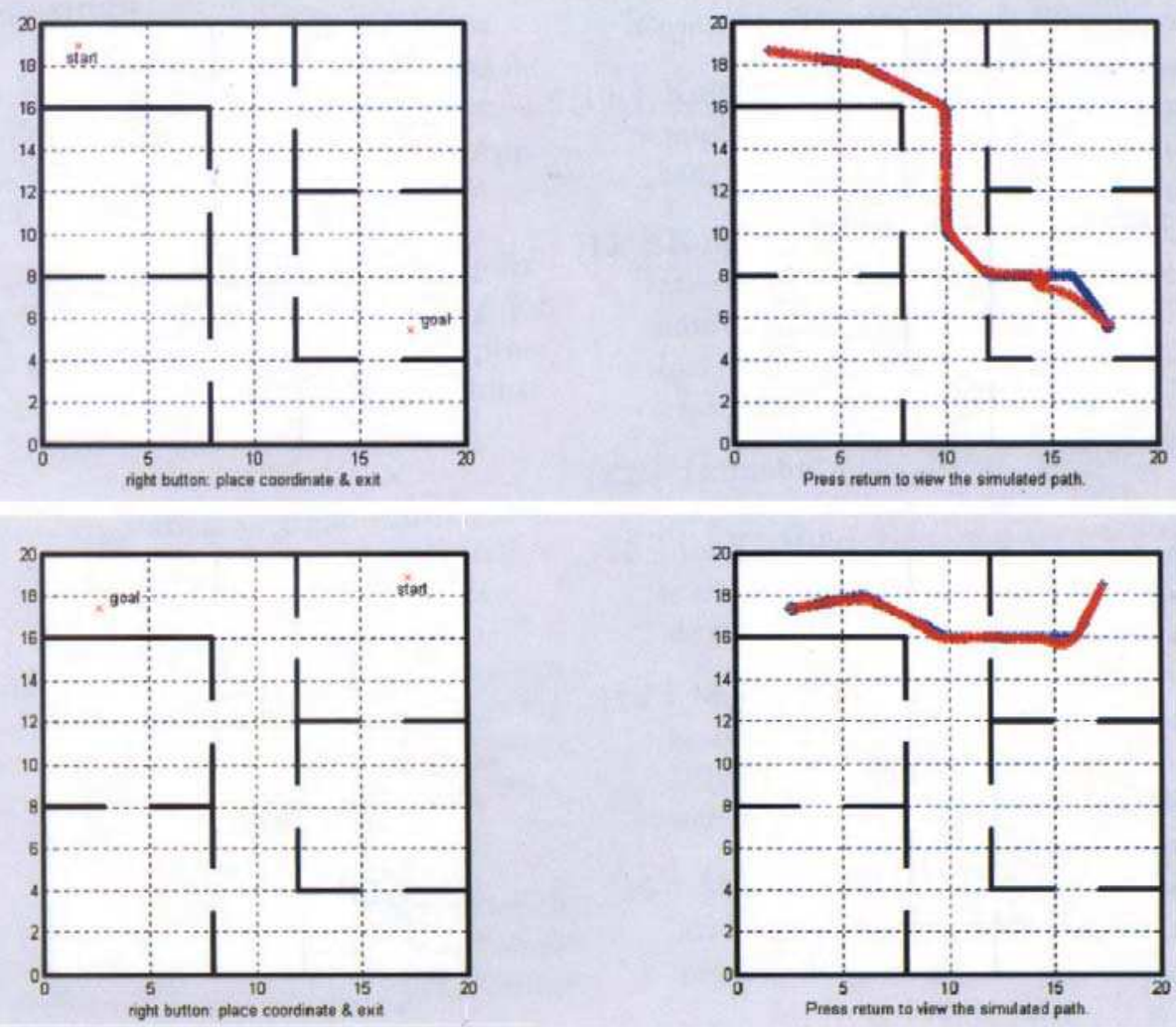

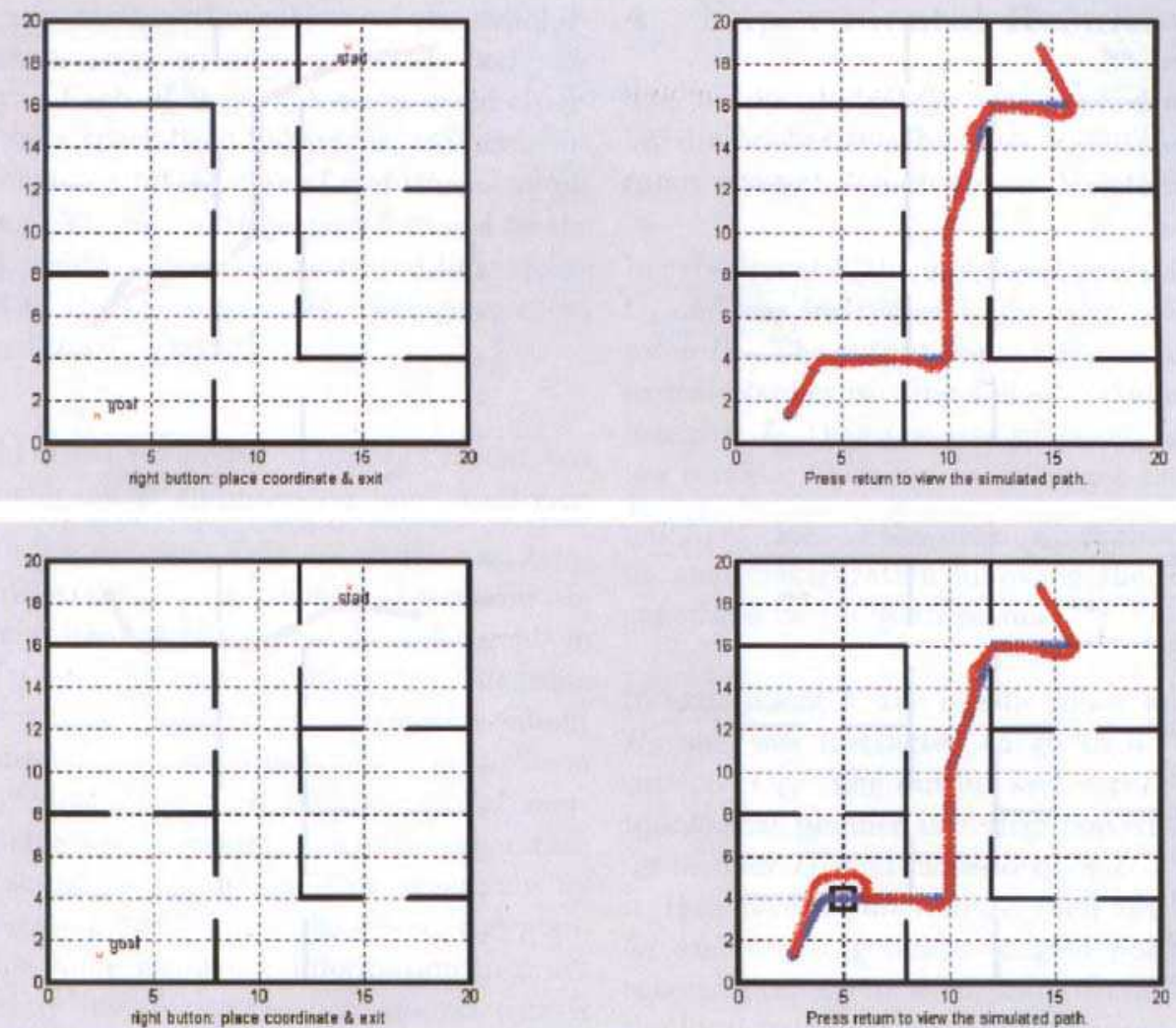

Figure 10. Illustration of mobile robot global path planning experiments.

In experiment 3, the mobile robot was in room $R_{1}$ and was instructed to go to an office book - case in room $R_{4}$. The output sequence / plan of the topological planner is : first traverse room $R_{1}$ up to door $D_{1}$, get-close-to door $D_{1}$, then cross it, then traverse corridor $C_{2}$ up to door $D_{7}$, then get-close-to door $D_{7}$, then cross it, then traverse room $R_{4}$, and finally get-close-to the bookcase. The robot moved to its goal configuration following the local paths generated by the path planner.

In experiment 4, experiment 3 was repeated. The mobile robot was in room $R_{1}$ and was instructed to go to an office bookcase in room $R_{4}$. The output sequence / plan of the topological planner is : first traverse room $R_{1}$ up to door $D_{1}$, get-close-to door $D_{1}$, then cross it, then traverse corridor $C_{2}$ up to door $D_{7}$, then get-close-to door $D_{7}$, then cross it, then traverse room $R_{4}$, and finally getclose-to the bookcase. The robot moved to its goal configuration following the local paths generated by the path planner. However, an unexpected obstacle occurred in room $R_{4}$ and the local reactive planner made the appropriate adjustments to the robot's motion in order to avoid the collision. 


\section{Conclusions and Directions for Research}

In this chapter the problem of collision - free path planning and navigation was studied for both robotic manipulators and omnidirectional mobile robots. A short review of the problem itself was provided along with a number of fundamental and recent references. The methodology followed was based on fuzzy logic and reasoning and a promising fuzzy path planner and navigator was developed, that can be applied to both industrial and mobile robots. This path planner / navigator has been tested by simulation in a variety of working scenarios with different obstacle constellations, both static and dynamic, providing each time a collision-free trajectory for the robotic manipulator. This local planning method has shown a robust and stable performance and the experimental results were very satisfactory. The difference between the presented approach and the existing obstacle avoidance techniques is that the proposed navigator considers only the nearest obstacle to decide upon the ro-

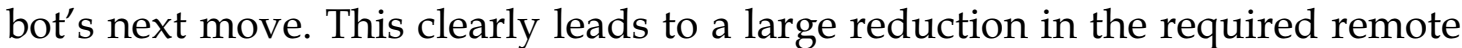
sensing and computations. A drawback is that, this reduction in information about the robot's environment, leads to an increased possibility of getting trapped in local minima. There may be routes leading to the goal that avoid the obstacles, which our navigator will not be able to find.

Concerning the mobile robots both local and global path planners were developed and tested. The local geometric information was encoded in a set of local sectors, whereas the global topological information was encoded in a network connecting these sectors. Each sector is a Cartesian representation, with its own reference system, that covers a limited area of the environment, like a room, a hall, or a corridor, and includes an approximate geometric description of the boundaries of the objects in the environment. The environment at the local level was represented by local metric maps connected to the topological network. In this way one can use maps that are not metrically consistent on the global scale although they are metrically consistent locally. This structure allows also the combination of abstract global reasoning and precise local geometric computations. To navigate in the environment, the robot uses, at the higher level of abstraction, the topological information to plan a sequence of sectors and gateways to traverse, and, to the lower level, uses the metric information in each sector to perform geometric path planning and navigation, and to locally move within the sector and to the next one. The system has shown a very stable and robust performance, providing each time the mobile robot with a collision free movement. The proposed fuzzy navigator is very fast and can be used in real time.

Future research may be devoted to the application of neural learning mechanisms applied on the fuzzy navigator providing an adaptive neurofuzzy planner (Topalov \& Tzafestas, 2001; Brown, 1994; Tzafestas \& Zavlangas, 1999; Tzafestas \& Zikidis, 2001; Wang, 1994; Stamou \& Tzafestas, 2000). The authors' 
group is also investigating the motion planning and control problem of mobile robots equipped with manipulator arms, i.e., mobile manipulators (Tzafestas \& Tzafestas, 2001; Bayle et al., 2003; Tzafestas et al., 2000; Erden et al., 2004). Other potential directions for future research needed in both industrial and non-industrial (e.g., service, medical) applications include multirobotic systems involving industrial manipulators, mobile robots and mobile manipulators, and the application of behavior-based techniques to both single robotic and multiple cooperating robots (Erden et al., 2004; Hsu \& Liu, 2005; Arai et al., 1999; Balch \& Arkin, 1998; Yamaguchi et al., 2001; Wang, 1991).

\section{References}

Althoefer, K. (1996). Fuzzy obstacle avoidance for robotic manipulators, Neural Network World, 6(2), 131-142

Althoefer, K. \& Fraser, D. A. (1996). Robotic manipulators amidst moving obstacles : Fuzzy - based obstacle avoidance, in Proc. of EUFIT '96 - The $4^{\text {th }}$ European Congress on Intelligent Techniques and Soft Computing, Aachen.

Arai, T.; Pagello, E. \& Parker, L. E. (1999). (Guest Editorial), IEEE Trans. on Robotics and Automation, 15(5), 818-828.

Balch, T. \& Arkin, R. C. (1998). Behavior - based formation control for multirobot teams, IEEE Trans. Robotics and Automation, 14(6), 926-939.

Bayle, B.; Renaud, M. \& Fourquet, J.-Y. (2003). Nonholonomic mobile manipulators: kinematics,velocities and redundancies, J. Intell. and Robotic Systems, 36(1), 45-63.

Beaufrere B. \& Zeghloul, S. (1995). A mobile robot navigation method for using a fuzzy based method : Simulation and experimental results, Intl. J. Robotics and Automation, 10(3), 106-113.

Belkhous, S.; Azzouz, A.; Saad, M.; Nerguizian, C. \& Nerguizian, V. (2005). A novel approach for mobile robot navigation with dynamic obstacles avoidance, J. Intell. and Robotic Systems, 44(3), 187-201.

Boem, H. R. \& Cho, H. S. (1995). A sensor-based navigation for a mobile robot using fuzzy - logic and reinforcement learning, IEEE Trans. Systems, Man and Cybernetics, 25(3), 464-477.

Brooks, R. A. (1986). A robust layered control system for a mobile robot, IEEE J. Robotics and Automation, 2(1), 14-23, 1986.

Brown, M. (1994). Neuro-Fuzzy Adaptive Modelling and Control, Prentice-Hall, UK. 
Dudek, G.; Jenkin, M.; Milios, E. \& Wilkes, D. (1991). Robotic exploration as graph construction, IEEE Trans. on Robotics and Automation, 7(6), 859-865.

ajjaji, A. El. (2004). A Ciocan and D. Hamad, Four wheel steering control by fuzzy approach, J. Intell. and Robotic Systems, 41(2-3), 141-156.

Erden, M. S.; Leblebicioglu, K. \& Halici, U. (2004). Multi - agent system based fuzzy controller design with genetic tuning for a mobile manipulator robot in the hand over task, J. Intell. and Robotic Systems, 39(3), 287-306.

Erdmann, M. \& Lozano-Perez, T. (1987). On Multiple Moving Obstacles, Algorithmica 2(4), 477-521.

Fibry, J. R. (1987). An Investigation into Reactive Planning in Complex Domains, Proc. AAAI Conference.

Fugimura, K. (1991). Motion Planning in Dynamic Environments, Springer, Tokyo.

Gat, E. (1991). Reliable Goal-Directed Reactive Control for Real - World Autonomous Mobile Robots, PhD Dissertation, Virginia Polytechnic Institute and state University.

Gil de Lamadrid, J. \& Gini, M. (1990). Path Tracking through Uncharted Moving Obstacles, IEEE Transactions on System, Man, and Cybernetics, 20(6), 1408-1422.

Griswold, N. C. \& Elan, J. (1990). Control of Mobile Robots in the Presence of Moving Obstacles, IEEE Transactions on Robotics and Automation 6(2).

Hsu, H. C.-H. \& Liu, A. (2005). Multiagent - based multi-team formation control for mobile robots, J. Intell. and Robotic Systems, 42(4), 337-360.

Ishikawa, S. (1995). A method of autonomous mobile robot navigation by using fuzzy control, Advanced Robotics, 9(1), 29-52.

Izumi, K. \& Watanabe, K. (2000). Fuzzy behavior - based control trained by module learning to acquire the adaptive behaviors of mobile robots, Mathem. and Computers in Simulation, 51(3/4), 233-243.

Jaitly, R.; Althoefer, K. \& Fraser, D. (1996). From vision to path planning : A neural-based implementation, Proc. $2^{\text {nd }}$ international conference on engineering applications of neural networks, 209-212, London, UK.

Jaitly, R. \& Fraser, D. A. (1996). Automated 3D object recognition and library entry system, Neural Network world, 6(2), 173-183.

Kamon, I. \& Rivlin, E. (1997). Sensory-based motion planning with global proofs, IEEE Trans. Robotics and Automation, 13(6).

Khatib, O. (1986). Real - time obstacle avoidance for manipulators and mobile robots, Internat. J. Robotics Res. 5(1), 90-98.

Kortenkamp, D. \& Weymouth, T. (1994). Topological mapping for mobile robots using combination of sonar and vision sensing, Proc. AAAI Conf., Menlo Park, CA, USA, 979-984.

Kosko, B. (1992). Neural Networks and Fuzzy Systems. A Dynamical Systems Approach to Machine Intelligence, Prentice - Hall, Englewood Cliffs, NJ, 2-3, 314-316. 
Kuipers, B. \& Levitt, T. (1988). Navigation and mapping in large scale space, AI Magaz., 9, 25-43.

Latombe, J. C. (1991). Robot Motion Planning, Kluwer, Boston.

Lozano-Perez, T. (1983). Spatial planning : A configuration space approach, IEEE Trans. Comput. 32(2), 108-120.

Mamdani, E. H. (1974). Applications of fuzzy algorithms for simple dynamic plant, Proc. IEE 121(12), 1585-1588.

Mamdani, E. H. \& Assilian, S. (1981). An experiment in linguistic synthesis with a fuzzy logic controller, in : Fuzzy Reasoning and its Applications, Academic Press, New York, 311-323.

Martinez, A.; Tunstel, E. \& Jamshidi, M. (1994). Fuzzy - logic based collision avoidance for a mobile robot, Robotica, 12 (6), 521-527.

Moustris, G. \& Tzafestas, S. G. (2005). A robust fuzzy logic path tracker for non-holonomic mobile robots, Intl. J. Artif. Intelligence Tools, 14(6), 935965.

Parhi, D. R. (2005). Navigation of mobile robots using fuzzy logic, J. Intell. and Robotic Systems, 42(3), 253-273.

Pedrycz, W. (1995). Fuzzy Sets Engineering, CRC Press, Boca Raton, FL, 3-7.

Ratering, S. \& Gini, M. (1995). Robot Navigation in a Known Environment with Unknown Obstacles, Autonomous Robots, 149-165.

Rigatos, G. G.; Tzafestas, C. S. \& Tzafestas, S. G. (2000). Mobile robot motion control in partially unknown environments using a sliding mode fuzzy controller, Robotics and Autonomous Systems, 33(1), 1-11.

Ryu, B. S. \& Yang, H. S. (1999). Integration of reactive behaviors and enhanced topological map for robust mobile robot navigation, IEEE Trans. on Systems, Man and Cybernetics, 29(5), 474-485.

Seraji, H. (1998). A unified approach to motion control of mobile manipulators, Intl. J. Robotics Research, 17(2), 107-118.

Seraji H. \& Howard, A. (2002). Behavior - based robot navigation on challenging terrain : A fuzzy logic approach, IEEE Trans. Robotics and Automation, 18(3), 308-321.

Stamou G. B. \& Tzafestas, S. G. (2000). Neural fuzzy relational systems with a new learning algorithm, Mathem. and Computers in Simulation, 51(3-4), 301314.

Sugeno, M. (1985). An Introductory Survey of Fuzzy Logic, Information Science, $36,59$.

Sugeno, M. \& Murakami, K. (1984). Fuzzy Parking Control of Model Car, Proc. $23^{\text {rd }}$ IEEE Conf. on Decision and Control, 902, Las Vegas, USA.

Sugeno, M. \& Nishida, M. (1985). Fuzzy Control of a Model Car, Fuzzy Sets and Systems, 16, 103-113.

Thrun, S. (1999). Learning metric-topological maps for indoor mobile robot navigation, Artificial Intelligence, 1, 21-71. 
Topalov, A. V. \& Tzafestas, S. G. (2001). Fuzzy - neural - genetic layered multi - agent reactive control of robotic soccer, In : D. Braha (ed.), Data Mining for Design and Manufacturing, Kluwer, Dordrecht / Boston, 417-422.

Tzafestas, S. G. (1999) (ed.), Advances in Intelligent Autonomous Systems, Kluwer, Dordrecht / Boston.

Tzafestas, S. G; Melfi, A. \& Krikochoritis, T. (2000). Modelling and Control of an Omnidirectional Mobile Manipulators, Proc. BASY'2000 : $4^{\text {th }}$ IEEE/IFIP Intl. Conf. on Inf. Technology for Balanced automation Systems, Berlin, Sept. 27-29.

Tzafestas, C. S. \& Tzafestas, S. G. (2001). Full-state modeling, motion planning and control of mobile manipulators, Studies in Informatics and Control, 10(2), 109-127.

Tzafestas, S. G. \& Zavlangas, P. G. (1999). Adaptive neuro-fuzzy navigation for industrial manipulators, Proc. 1999, ICIMS-NOE Advanced Summer Institute : Production Management, Control and Supervision (ASI '99), Leuven, Belgium.

Tzafestas S. G. \& Zikidis, K. C. (2001). NeuroFast : On - line Neuro-Fuzzy ART-based Structure and Parameter Learning TSK Model, IEEE Trans. Syst., Man, and Cybernetics, Part B : Cybernetics, 31(5).

Tzafestas, S. G. \& Venetsanopoulos, A. N. (1991) (eds.), Fuzzy Reasoning in Information, Decision and Control Systems, Kluwer, Dordrecht / Boston, 1994.

Wang, P. K. C. (1991). Navigation strategies for multiple autonomous mobile robots moving in formation, J. Robotic Systems, 8(2), 177-195.

Wang, L. X. (1994). Adaptive Fuzzy Sets and Control : Design and Stability Analysis, Prentice Hall Int., U.K.

Watanabe, K.; Izumi, K.; Maki, J. \& Fujimoto, K. (2005). A fuzzy behavior based control for mobile robots using adaptive fusion units, J. Intell. and Robotic Systems, 42(1), 42-27.

Watanabe, K.; Tang, J.; Nakamura, M.; Koga, S. \& Fukuda, T. (1996). A fuzzy Gaussian neural network and its application to mobile robot control, IEEE Trans. Control Systems Technol., 4(2), 193-199.

Yamaguchi, H.; Arai, T. \& Beni, G. (2001). A distributed control scheme for multiple robotic vehicles to make group formations, Robotics Auton. Syst., 36(4), 125-147.

Yen, J. \& Pflunger, N. (1992). A Fuzzy Logic Based Robot Navigation System, Proc. AAAI Fall Symposium on Mobile Robot Navigation, 195-199, Boston / MA, USA.

Zadeh, L. (1965). Fuzzy sets, Inform. E Control, 8, 338-353.

Zadeh, L. (1973). Outline of a new approach to the analysis of complex systems and decision processes, IEEE Trans. Systems Man Cybernetics, 1, 28-44.

Zavlangas, P. G. \& Tzafestas, S. G. (2000). Industrial robot navigation and obstacle avoidance employing fuzzy logic, J. Intell. and Robotic Systems, 27(12), 85-97. 
Zavlangas, P. G. \& Tzafestas, S. G. (2001). Integrated fuzzy global path following and obstacle avoidance for mobile robots, Systems Science, 27(4), 85-96. Zavlangas, P. G. \& Tzafestas, S. G. (2002). Integration of topological and metric maps for indoor mobile robot path planning and navigation, Image Processing and Communications, 8(1), 55-64. 


\section{Industrial Robotics: Theory, Modelling and Control \\ Edited by Sam Cubero}

ISBN 3-86611-285-8

Hard cover, 964 pages

Publisher Pro Literatur Verlag, Germany / ARS, Austria

Published online 01, December, 2006

Published in print edition December, 2006

This book covers a wide range of topics relating to advanced industrial robotics, sensors and automation technologies. Although being highly technical and complex in nature, the papers presented in this book represent some of the latest cutting edge technologies and advancements in industrial robotics technology. This book covers topics such as networking, properties of manipulators, forward and inverse robot arm kinematics, motion path-planning, machine vision and many other practical topics too numerous to list here. The authors and editor of this book wish to inspire people, especially young ones, to get involved with robotic and mechatronic engineering technology and to develop new and exciting practical applications, perhaps using the ideas and concepts presented herein.

\section{How to reference}

In order to correctly reference this scholarly work, feel free to copy and paste the following:

Tzafestas S. G. and Zavlangas P. (2006). Industrial and Mobile Robot Collision-Free Motion Planning Using Fuzzy Logic Algorithms, Industrial Robotics: Theory, Modelling and Control, Sam Cubero (Ed.), ISBN: 386611-285-8, InTech, Available from:

http://www.intechopen.com/books/industrial_robotics_theory_modelling_and_control/industrial_and_mobile_ro bot_collision-free_motion_planning_using_fuzzy_logic_algorithms

\section{INTECH}

open science | open minds

\section{InTech Europe}

University Campus STeP Ri

Slavka Krautzeka 83/A

51000 Rijeka, Croatia

Phone: +385 (51) 770447

Fax: $+385(51) 686166$

www.intechopen.com

\section{InTech China}

Unit 405, Office Block, Hotel Equatorial Shanghai

No.65, Yan An Road (West), Shanghai, 200040, China

中国上海市延安西路65号上海国际贵都大饭店办公楼 405 单元

Phone: +86-21-62489820

Fax: +86-21-62489821 
(C) 2006 The Author(s). Licensee IntechOpen. This chapter is distributed under the terms of the Creative Commons Attribution-NonCommercial-ShareAlike-3.0 License, which permits use, distribution and reproduction for non-commercial purposes, provided the original is properly cited and derivative works building on this content are distributed under the same license. 